\title{
Glucose-based spiro-heterocycles as potent inhibitors of glycogen phosphorylase
}

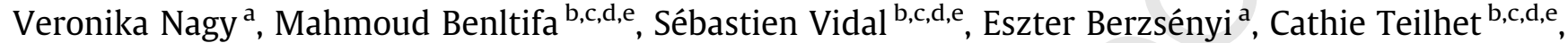 \\ Katalin Czifrák ${ }^{a}$, Gyula Batta ${ }^{\mathrm{f}}$, Tibor Docsa ${ }^{\mathrm{g}}$, Pál Gergely ${ }^{\mathrm{h}}$, László Somsák ${ }^{\mathrm{a}, *}$, Jean-Pierre Praly ${ }^{\mathrm{b}, c, \mathrm{~d}, \mathrm{e}, *}$ \\ a Department of Organic Chemistry, University of Debrecen, POB 20, H-4010 Debrecen, Hungary \\ ${ }^{\mathrm{b}}$ Université de Lyon, Institut de Chimie et Biochimie Moléculaires et Supramoléculaires associé au CNRS, UMR 5246, Laboratoire de Chimie Organique 2, \\ Bâtiment Curien, 43 Boulevard du 11 Novembre 1918, F-69622 Villeurbanne, France \\ ${ }^{\mathrm{C}}$ Université Lyon 1, F-69622 Villeurbanne, France \\ 10 d CNRS, UMR5246, Institut de Chimie et Biochimie Moléculaires et Supramoléculaires (ICBMS), Laboratoire de Chimie Organique 2, Bâtiment Curien, \\ 43 Boulevard du 11 Novembre 1918, F-69622 Villeurbanne, France \\ e CPE-Lyon, F-69616 Villeurbanne, France \\ ${ }^{\mathrm{f}}$ Department of Biochemistry, University of Debrecen, Egyetem tér 1, H-4032 Debrecen, Hungary \\ ${ }^{\mathrm{g}}$ Cell Biology and Signaling Research Group of the Hungarian Academy of Sciences at the Department of Medical Chemistry, Medical and Health Science Centre, \\ University of Debrecen, Egyetem tér 1, H-4032 Debrecen, Hungary \\ ${ }^{\mathrm{h}}$ Department of Medical Chemistry, Medical and Health Science Centre, University of Debrecen, Egyetem tér 1, H-4032 Debrecen, Hungary
}

\section{A R T I C L E I N F O}

Article history:

Received 12 January 2009

Revised 25 May 2009

Accepted 31 May 2009

Available online $\mathrm{xxxx}$

\section{Keywords:}

Glucosyl-hydroximothioates

Spiro-cyclization

Spiro-compounds

30 Glucosylidene-spirooxathiazoles

Inhibitors

Glycogen phosphorylase

Carbohydrates

Glycomimics

\begin{abstract}
A B S T R A C T
Glucopyranosylidene-spiro-1,4,2-oxathiazoles were prepared in high yields by NBS-mediated spiro-cyclization of the corresponding glucosyl-hydroximothioates. In an effort to synthesize analogous glucopyranosylidene-spiro-1,2,4-oxadiazolines, with a nitrogen atom instead of the sulphur, attempted cyclizations resulted in aromatization of the heterocycle with opening of the pyranosyl ring. Enzymatic measurements showed that some of the glucose-based inhibitors were active in the micromolar range. The 2-naphthyl-substituted 1,4,2-oxathiazole displayed the best inhibition against $\operatorname{RMGPb}\left(K_{\mathrm{i}}=160 \mathrm{nM}\right)$, among glucose-based inhibitors known to date.
\end{abstract}

(C) 2009 Published by Elsevier Ltd.

\section{Introduction}

Type 2 diabetes mellitus ${ }^{1}$ is currently estimated to affect more than $5 \%$ of the adult population in Western societies, and its incidence is expected to increase considerably in the future. ${ }^{2,3}$ This is in particular due to the dramatic increase in obesity even among young adults and children. ${ }^{4-6}$ Striking enhancements in the prevalence of the disease is predicted for the developing countries of Africa, Asia, and South America. ${ }^{2}$ Current preventive and therapeutic strategies do not achieve adequate control of blood glucose to diminish chronic morbidity, and there is a need to develop novel healthcare interventions to address this substantial biomedical challenge. ${ }^{7}$

\footnotetext{
* Corresponding authors. Tel.: +33 4724311 61; fax: +334 72448349 (J.-P.P.)

E-mail address: jean-pierre.praly@univ-lyon1.fr (J.-P. Praly).
}

Hepatic glucose output is elevated in type 2 diabetic patients and current evidence indicates that glycogenolysis (release of monomeric glucose from the glycogen polymer storage form) is an important contributor to the abnormally high production of glucose by the liver. Glycogen phosphorylase (GP) is the rate limiting enzyme in the liver responsible for glycogen breakdown to produce glucose and related metabolites for energy supply. ${ }^{8}$ Due to its key role in modulation of glycogen metabolism, pharmacological inhibition of GP has been regarded as an effective therapeutic approach to treating type 2 diabetes. ${ }^{9-11}$

Several types of compounds for inhibition of GP have been reported, ${ }^{10-13}$ and among them derivatives of D-glucose represent, as ligands of the catalytic site, the most populated class (Chart 1). ${ }^{12-15}$ There are three mammalian GP isoenzymes termed as 'brain', 'muscle', or 'liver' GP depending on the tissue in which they are preferentially expressed. All isoenzymes can be converted from the inactive form $(\mathrm{GPb})$ into the active $\mathrm{GPa}$ form through the phos- 


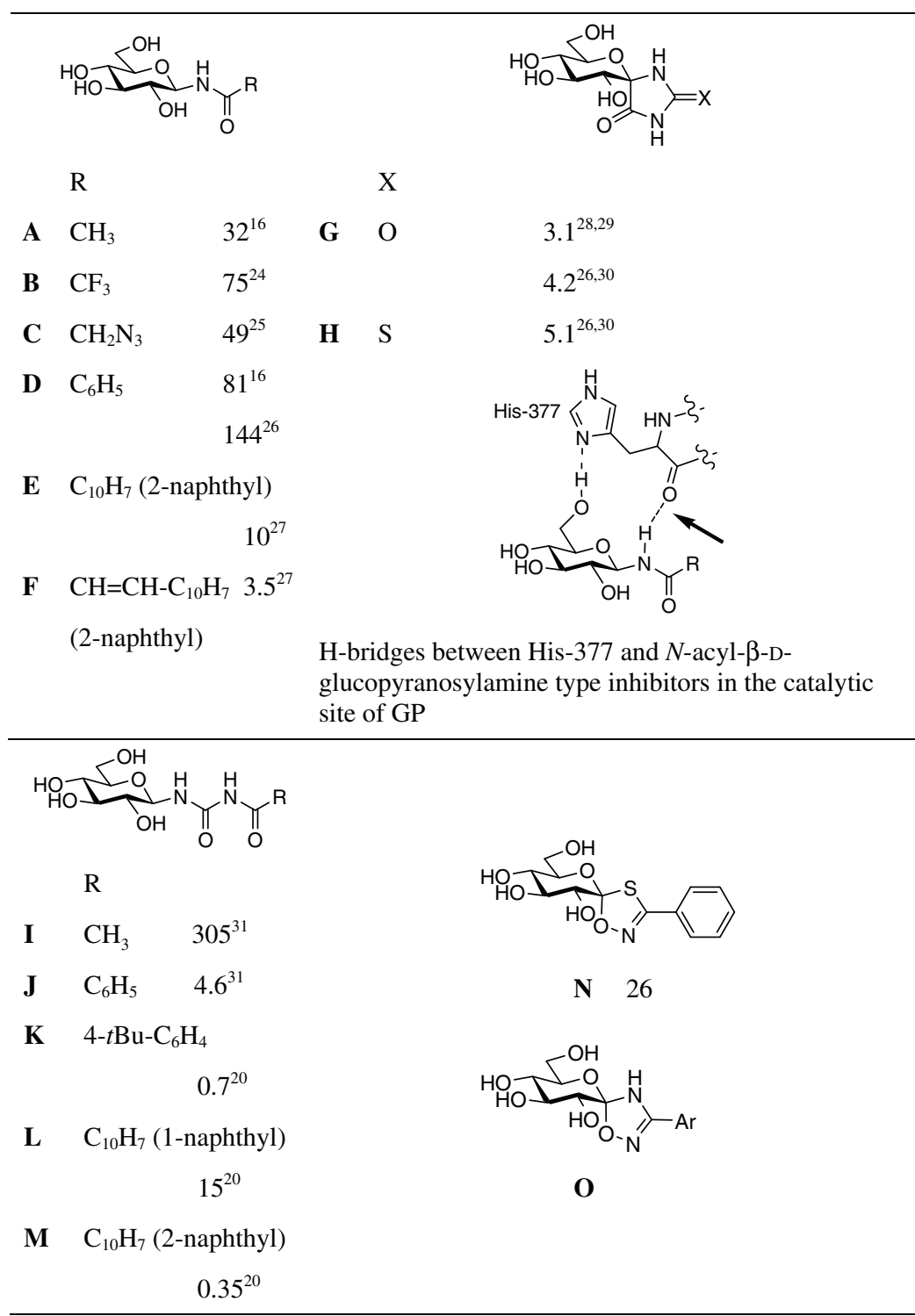

Chart 1. Inhibitory constants $\left(K_{\mathrm{i}}[\mu \mathrm{M}]\right)$ for some representative inhibitors of rabbit muscle glycogen phosphorylase. ${ }^{24,25,27}$

phorylation of Ser-14. Isoforms of GP can be obtained from rabbit squeletal muscles (RMGPa/RMGPb) or human liver (HLGPa/ HLGP $b$ ). Because of a $~ 80 \%$ homology between the liver and muscle isoforms of GP, it is a general practice to use the more readily available RMGP (most frequently in the unphosphorylated $b$ form) for preliminary kinetic studies (Chart 1 ), even though, for in vivo experiments, the existence of isoforms raises the question of the selectivity of inhibition. ${ }^{13}$ The functional form of GP is a homodimer having a C-2 symmetry. The catalytic site of GP is buried at the centre of the monomers, and is accessible to the bulk solvent through a $15 \AA$ A long channel. The site has been extensively investigated with glucose analogue inhibitors which promote the less active $T$ (tensed) state through stabilisation of the closed position of the 280 s loop. Upon transition from the $\mathrm{T}$ state to the $\mathrm{R}$ (relaxed) state, the 280s loop becomes disordered and displaced, resulting in the activation of the enzyme. Crystallographic investigations have shown the existence of an empty pocket in the direction of the $\beta$-anomeric substituent of $\mathrm{D}$-glucopyranose, lined by both polar and non-polar group, and named the $\beta$-channel. ${ }^{10}$

$N$-Acetyl- $\beta$-D-glucopyranosylamine ${ }^{16} \mathbf{A}$ was one of the first efficient glucose analogue inhibitors of GP. A large array of compounds with the $N$-acyl- $\beta$-D-glucopyranosylamine structure (e.g., B-F) was synthesized, tested, and also investigated by X-ray crystallography. ${ }^{10}$ These studies revealed that a $\mathrm{H}$-bridge between the amide $\mathrm{NH}$ and main chain $\mathrm{CO}$ of His377 (Chart 1) significantly contributed to the binding. This H-bridge was also observed in GP crystal complexes of the low micromolar inhibitor spiro-hydantoins $\mathbf{G}^{17}$ and $\mathbf{H}^{18}$ and was, thereby, considered to be an essential feature for strong inhibition. The rigidity of the spiro-bicyclic structure of the latter compounds with a pyranoid sugar and a five-membered heterocyclic ring was also considered to be a decisive factor for the efficiency. ${ }^{17}$ On the other hand, $N$ - $\beta$-D-glucopyranosyl- $N$-acyl ur-

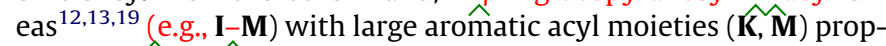
erly oriented so âs to interact with the side chains of the so-called $\beta$-channel next to the catalytic site, exhibit nanomolar inhibition $^{12,13,20}$ despite the lack of the H-bond mentioned above. Therefore, interactions in the so called $\beta$-channel of the enzyme must play a more important role than that particular H-bond.

Based on the binding peculiarities of these inhibitors, we envisaged to prepare compounds which might unify the structural features of the best inhibitors, that is, spiro junction between the anomeric carbon of D-glucopyranose and a five-membered hetero- 
cycle, as well as a large aromatic substituent capable of interactions in the $\beta$-channel. This design was supported by the finding that spiro-oxathiazole $\mathbf{N}$, available from earlier synthetic studies, $^{21,22}$ proved to be a micromolar inhibitor. Therefore, synthesis of further compounds of this series was carried out ${ }^{23}$ and, in order to facilitate the formation of an additional H-bond, preparation of spiro-oxadiazolines of type $\mathbf{0}$ was also attempted.

\section{Results and discussion}

\subsection{Preparation of glucosyl-hydroximothioates}

The R-glucosyl hydroximothioates $1 \mathbf{a}-\mathbf{j}$ were prepared from per- $O$-acetylated 1 -thio- $\beta$-D-glucopyranose and in situ formed nitrile oxides used in slight excess (1.2 equiv), following a procedure reported previously by Rollin ${ }^{32}$ (Scheme 1 , Table 1 ). The synthesis of $1 \mathbf{i}(\mathrm{Ar}=4$-pyridyl $)$ was optimized by increasing the amount of 4pyridyl-hydroximoyl chloride used for producing the corresponding nitrile oxide with yields growing as follows: 6\% (1.4 equiv), $48 \%$ ( 3.8 equiv), $78 \%$ (5 equiv). The prepared hydroximothioates which appeared to be stable under normal conditions were deacetylated by standard procedures $\left(\mathrm{NaOMe} / \mathrm{MeOH}\right.$ or $\mathrm{MeOH} / \mathrm{H}_{2} \mathrm{O} / \mathrm{Et}_{3} \mathrm{~N}$ ) to afford the corresponding unprotected products in high yields (Scheme 1).

In an attempt to prepare molecules susceptible to bind more tightly at the active site of GP through non covalent interactions involving a polar group near the anomeric center of the ligand, we tried to oxidize compound $\mathbf{1 i}(\mathrm{Ar}=4$-pyridyl) with m-CPBA, as applied earlier for the synthesis of glucosyl-phenyl-sulfoxides. ${ }^{33}$ The oxidation was not selective, since the reaction mixture was shown by TLC to contain a mobile compound, and two major polar products. One of them was shown to be the $\mathrm{N}$-oxide $\mathbf{1 j}$ ( $\mathrm{Ar}=4$-pyridyl- $N$-oxide), isolated in ca $30 \%$ yield.

\subsection{NBS-mediated cyclization of hydroximothioates}

The spiro-cyclization of phenyl hydroximothioate 1 a proceeded in $\mathrm{CHCl}_{3}$ with the same selectivity as already observed in $\mathrm{CCl}_{4}{ }^{22,33}$ affording a 5:1 mixture of the $(1 S) /(1 R)$-epimers of $\mathbf{2 a}$ (Scheme 1). The reaction of hydroximothioates $\mathbf{1 b}, \mathbf{c}, \mathbf{e}, \mathbf{g}, \mathbf{h}$ with $\mathrm{N}$-bromosuccinimide (NBS) in freshly distilled $\mathrm{CCl}_{4}$ or $\mathrm{CHCl}_{3}$ was stopped when TLC monitoring indicated complete conversion of the substrates, ca $45 \mathrm{~min}$ at reflux under irradiation with a tungsten lamp $(60 \mathrm{~W})$. This afforded the spiro-bicycles $\mathbf{3 b}, \mathbf{c}, \mathbf{e}, \mathbf{g}, \mathbf{h}$ as a mixture of epimers, with the predominance of the (1S)-configured compounds. Separation by flash column chromatography afforded first the pure (1S)-epimers. The fractions collected next contained epimeric mixtures whose composition was estimated by ${ }^{1} \mathrm{H}$ NMR to permit calculation of the yield for each epimer (Table 1). This

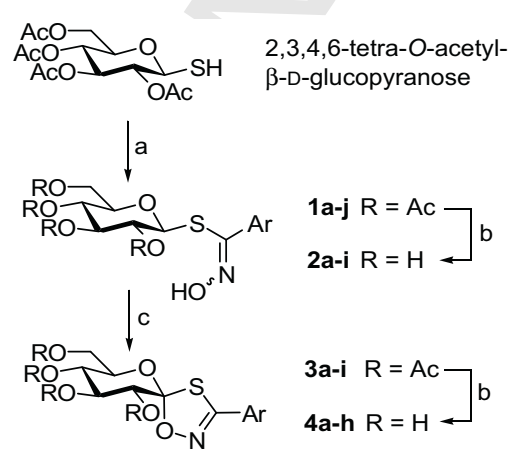

Scheme 1. Synthesis of D-glucopyranosylidene-spiro-oxathiazoles. Reagents and conditions: (a) $\mathrm{ArC}(\mathrm{Cl})=\mathrm{NOH}, \mathrm{Et}_{3} \mathrm{~N}$; (b) $\mathrm{NaOMe}, \mathrm{MeOH}$, or $\mathrm{MeOH}, \mathrm{Et}_{3} \mathrm{~N}, \mathrm{H}_{2} \mathrm{O}$, rt; (c) $\mathrm{NBS}, h v, 45 \mathrm{~min}$, in refluxing $\mathrm{CCl}_{4}$ or $\mathrm{CHCl}_{3}$. spiro-cyclization is believed to proceed through a 1,5-hydrogen transfer to alkoxyl radicals to create predominantly $\alpha$ oriented $\mathrm{C}-\mathrm{O}$ bonds. ${ }^{34,35}$ Spiro-cyclization of $\mathbf{1 f}$ was not selective under the applied conditions (heating for $1 \mathrm{~h}$ with a lamp), yielding a multicomponent mixture. Among the four fractions obtained by column chromatography, only the third one was pure enough to get exploitable NMR data corresponding probably to the $(1 R)-\mathbf{3 f}$ spiro compound, because of the observed pattern of the proton signals resonating at $\delta=5.59,5.10,5.27$ and $4.10 \mathrm{ppm}$ for protons $\mathrm{H}$ 2, $\mathrm{H}-3, \mathrm{H}-4$ and $\mathrm{H}-5$, respectively (in $\mathrm{CDCl}_{3}$ ).

Treatment of 1i with NBS ( 2 e्रquiv) in boiling chloroform for $30 \mathrm{~min}$ resulted in the formation of two major and two minor compounds (TLC). Column chromatography afforded only one homogeneous fraction identified as $(1 R)-3 \mathbf{i}(30 \%)$, based on NMR data and the fact that $(1 R)$-epimers were found to be significantly more dextrorotatory than the (1S)-epimers. ${ }^{22,33}$ Since spiro-cyclization afforded in this case a higher proportion of the $(1 R)$-epimer, we wondered whether this was due to the basic properties of pyridine. Therefore, the spiro-cyclization of $\mathbf{1 a}(\mathrm{Ar}=$ phenyl $)$ was carried out in the presence of DBU (1 e्रquiv) affording $2 \mathbf{a}$ (34\% yield) in a 1:1 $(1 S) /(1 R)$ ratio after repeated column chromatographies. As compared to the selectivity normally observed (60\% yield, 5:1 (1S)/ $(1 R)$ ratio), this change suggested a variation along the possible reaction pathways.

The Zemplén deacetylation was achieved in high yields to obtain the desired spiro-oxathiazoles $\mathbf{4 b}, \mathbf{c}, \mathbf{e}, \mathbf{g}, \mathbf{h}$. The final products had a very poor solubility in $\mathrm{H}_{2} \mathrm{O}, \mathrm{MeOH}$, EtOH (less than $0.1 \mathrm{mg}$ / $\mathrm{mL}$ ) but were soluble in DMF or DMSO.

\subsection{Attempted preparations of glucosylidene-spiro-1,2,4- oxadiazolines}

The synthesis of glucosylidene-spiro-1,2,4-oxadiazolines was planned as nitrogen-containing spiro analogues by applying again a NBS-mediated cyclization reaction to $N$ - $\beta$-D-glucosyl-amidoximes 8 (Scheme 2). A detailed retrosynthetic analysis and the preparation of the amidoximes ${ }^{36}$ are presented in the Supporting Information. Preparation of the required amidoxime 8 from per$\mathrm{O}$-acetylated glucopyranosyl bromide $\mathbf{5}$ or glucopyranosylamine $\mathbf{6}$ failed. Fortunately, in the presence of trimethylphosphine, acetylated $\beta$-D-glucopyranosyl azide $\mathbf{7}$ was transformed into the corresponding phosphinimine derivative ${ }^{37}$ which reacted readily with hydroximoyl chlorides ${ }^{38-40}$ and gave the target molecules $\mathbf{8 a - c , h}$. The NMR spectra of the crude products indicated the presence of two species ( $\sim 1: 10$ ratio) assumed to be the $E$ and $Z$-configured isomers. Due to the poor stability of some of these compounds, only $\mathbf{8 b}$ and $\mathbf{8 c}$ could be deacetylated by the Zemplén method to give $\mathbf{9 b}$ and $\mathbf{9 c}$.

The oxidative photocyclization of 4-nitrobenzamidoxime $\mathbf{8 b}$ was carried out under conditions similar to those applied in the synthesis of spiro-oxathiazoles 3. A chloroform solution of $\mathbf{8 b}$ and NBS was irradiated with a heating lamp (60 or $375 \mathrm{~W}$ ). NBS (4 equiv, twice as much as for the oxathiazoles) had to be added at once or portion wise to completely transform the starting material. Three experiments afforded mixtures which did not contain the expected spiro-molecule IV (Scheme 2) but separation by column chromatography afforded $\mathbf{1 0 b}, \mathbf{1 1 b}$, and $\mathbf{1 2 b}$ in variable proportions (Table 2). Nitroso compound 10b should result from $\mathbf{8 b}$ by oxidation without cyclization. However, formation of $\mathbf{1 1 b}$ and 12b clearly indicated that spiro-cyclization must have occurred followed by ring opening of the pyranosyl ring to allow aromatization of the oxadiazole ring. Compound $\mathbf{1 2 b}$ displayed in addition a carbonyl due to oxidation of the liberated hydroxyl at C-5. The product distribution was highly dependent on the applied conditions which were not optimized, although it was possible to obtain 12b as a single product. 
Table 1

Preparation of hydroximothioates 1-2 and glucosylidene-spiro-oxathiazoles 3-4

\begin{tabular}{|c|c|c|c|c|c|}
\hline \multirow{2}{*}{$\frac{\mathrm{Ar}=}{\mathrm{Ph}}$} & \multicolumn{5}{|c|}{ Compound/yield ${ }^{\mathrm{a}}(\%)$} \\
\hline & $\mathbf{2 a} / 9432$ & 1a/9032 & $(1 S)-\mathbf{3 a} / 46$ & $(1 R)-3 \mathbf{a} / 11$ & $(1 S)-\mathbf{4 a} / 90$ \\
\hline$p-\mathrm{NO} 2 \mathrm{Ph}$ & $\mathbf{2 b} / 9132$ & 1b/6032 & $(1 S)-\mathbf{3 b} / 33$ & $(1 R)-\mathbf{3 b} / 16$ & $(1 S)-\mathbf{4 b} / 71$ \\
\hline$p$-CNPh & & & $(1 S)-3 c / 15$ & $(1 R)-3 \mathbf{c} / 7$ & $(1 S)-\mathbf{4 c} / 84$ \\
\hline p-FPh & 2d/9532 & 1d/6532 & $(1 S)-\mathbf{3 d} / 3022$ & $(1 R)-\mathbf{3 d} / 1622$ & $(1 S)-\mathbf{4 d} / 79$ \\
\hline p-MeOPh & $\mathbf{2 e / 9 7 3 2}$ & 1e/7132 & $(1 S)-3 \mathbf{e} / 69$ & $(1 R)-3 \mathbf{e} / 17$ & $(1 S)-\mathbf{4 e} / 79$ \\
\hline 3,4,5-triMeOPh & & 1f $/ 85$ & Unselective reaction & & \\
\hline p-PhPh & & & $(1 S)-3 g / 61$ & $(1 R)-3 g / 18$ & $(1 S)-4 g / 97$ \\
\hline 2-Naphthyl & & & $(1 S)-\mathbf{3 h} / 36$ & $(1 R)-3 \mathbf{h} / 16$ & $(1 S)-\mathbf{4 h} / 94$ \\
\hline 4-Pyridyl & $2 \mathbf{i} / 90$ & $\mathbf{1 i} / 78$ & $(1 S)-\mathbf{3 i} / 0$ & $(1 R)-3 \mathbf{3} / 30$ & $(1 R)-4 \mathbf{i} / 95$ \\
\hline 4-Pyridyl- $N$-oxide & & $\mathbf{1 j} / 30$ & & & \\
\hline
\end{tabular}

${ }^{\text {a }}$ Calculated yields given in italics take into account the ratio of anomers estimated by ${ }^{1} \mathrm{H}$ NMR in mixed fractions after purification.

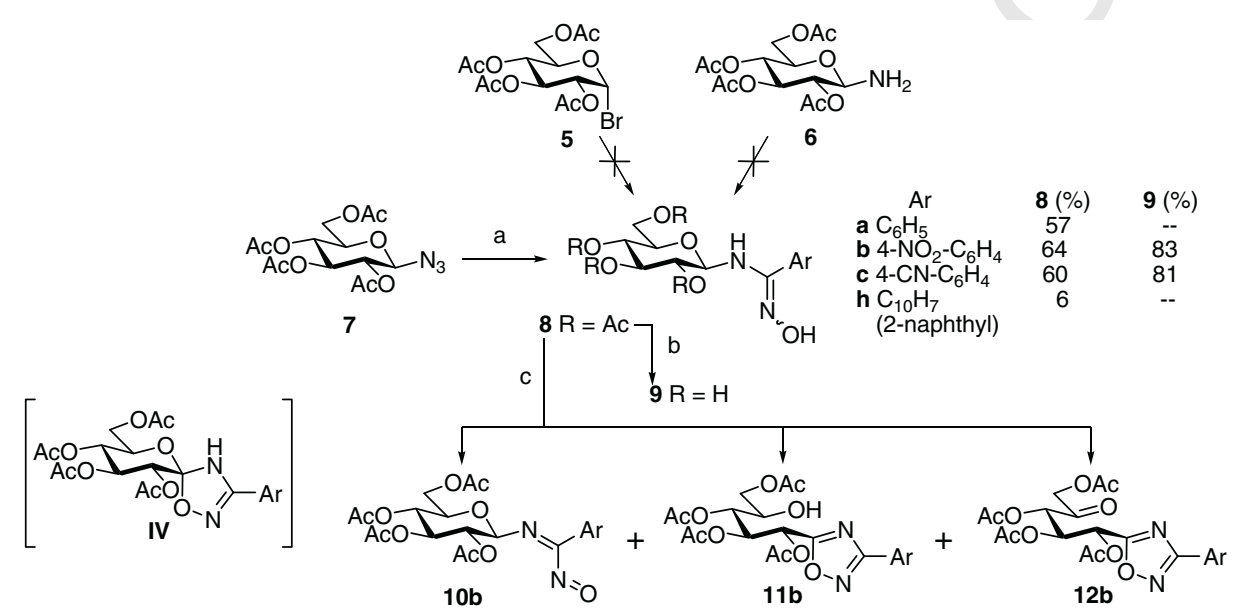

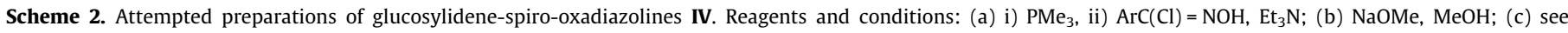
conditions in Table 2 .

Table 2

Photolysis of $\mathbf{8 b}$ under various conditions

\begin{tabular}{lllll}
\hline Lamp (W) & NBS (equiv) & Yield of 10b $(\%)$ & Yield of 11b $(\%)$ & Yield of 12b $(\%)$ \\
\hline 60 & 4 & 31 & 0 & $\sim 3$ \\
60 & $4 \times 1$ & 0 & 0 & 45 \\
375 & 4 & 0 & 20 & 10 \\
\hline
\end{tabular}

1,3-Dipolar cycloaddition of nitrile oxides to benzyl-protected glucosyl-hydroximolactone $13^{41,42}$ was envisaged as another approach to analogous glycopyranosylidene-spiro-1,2,4-oxadiazolines. Cycloadditions with aryl nitrile oxides derived from $\alpha$ chloro-benzaldoxime, $\alpha$-chloro- 4 -methoxybenzaldoxime, and $\alpha$ chloro-4-nitrobenzaldoxime proved to be disappointing since $\mathbf{1 3}$ underwent partial conversion after $24 \mathrm{~h}$ in boiling toluene in the presence of 8 equiv of 4-methoxybenzonitrile oxide. Among the isolated products (Scheme 3), we could identify the $\mathrm{N}$-oxide dimer $\mathbf{V}^{43}$ deriving from the nitrile oxide used and $\mathbf{1 5}$ (13\% isolated yield). Based on the conversion of $\mathbf{1 3}$, the calculated yield for $\mathbf{1 5}$ was $22 \%$. The low reactivity of $\mathbf{1 3}$ was confirmed by attempted reactions with either trimethylsilyldiazomethane in refluxing dioxane, or with bromonitrile oxide, which resulted only in slow and unselective transformations. Isolation of $\mathbf{V}\left(m / z=299.1[\mathrm{M}+\mathrm{H}]^{+}\right)$proved the existence, in the reaction mixture, of the 4-methoxybenzonitrile oxide which reacted slowly with 13. Mass spectrometry (ESI) confirmed that cycloaddition took place but it was not appropriate to discriminate between structures 14 or 15 which both might correspond to the observed ions $\left.(m / z=703[\mathrm{M}+\mathrm{H}]]^{+}\right) \cdot{ }^{13} \mathrm{C}$ NMR spectroscopy indicated chemical shifts at $140.8 \mathrm{ppm}$
$\left(\mathrm{N}_{2} \mathrm{CAr}\right)$ and $154.2 \mathrm{ppm}(\mathrm{NCO})$ characteristic of the oxadiazole $\mathrm{N}$ oxide ring. ${ }^{44}$ Formation of an aromatic oxadiazole ring with opening of the pyrane ring was in analogy with our previous observations from attempted spiro-cyclization of benzamidoximes.

\subsection{Inhibition of glycogen phosphorylase}

Enzymatic tests (Table 3) with deprotected hydroximothioates $\mathbf{2}$, benzamidoximes $\mathbf{9 b}, \mathbf{c}$, and the $(1 R)$-configured spiro-oxathiazole (1R)-4i showed no significant inhibition of rabbit muscle glycogen phosphorylase (RMGP) $b$. In contrast, several (1S)-configured spirocompounds (1S)-4 showed inhibition in the low micromolar range, except for compounds (1S)-4b,c having polar substituents (4-nitro and 4-cyano, respectively). The most efficient was the 2-naphthyl derivative (1S)-4h with a nanomolar $K_{\mathrm{i}}$ which appears to be the best glucose-based inhibitor known at the moment. This shows conclusively that a rigid spiro-bicyclic structure, attached to a properly oriented large apolar aromatic group as the 2-naphthyl are favorable structural features for strong binding at the catalytic site of GP. Further exploitation of these findings is in progress for the design of more potent inhibitors.

\section{Conclusion}

The recent identification of glycogen phosphorylase as a biological target for the treatment of hyperglycemia led to the design of a large collection of glucose-based inhibitors displaying inhibitory activities in the micromolar range. ${ }^{10,12-15}$ Among them, the glucopyranosylidene-spiro-hydantoins ${ }^{28-30}$ and $N$-acyl- $N$ - $\beta$-D-glucopyranosyl urea ${ }^{20,31}$ derivatives were shown to be the best inhibitors. 


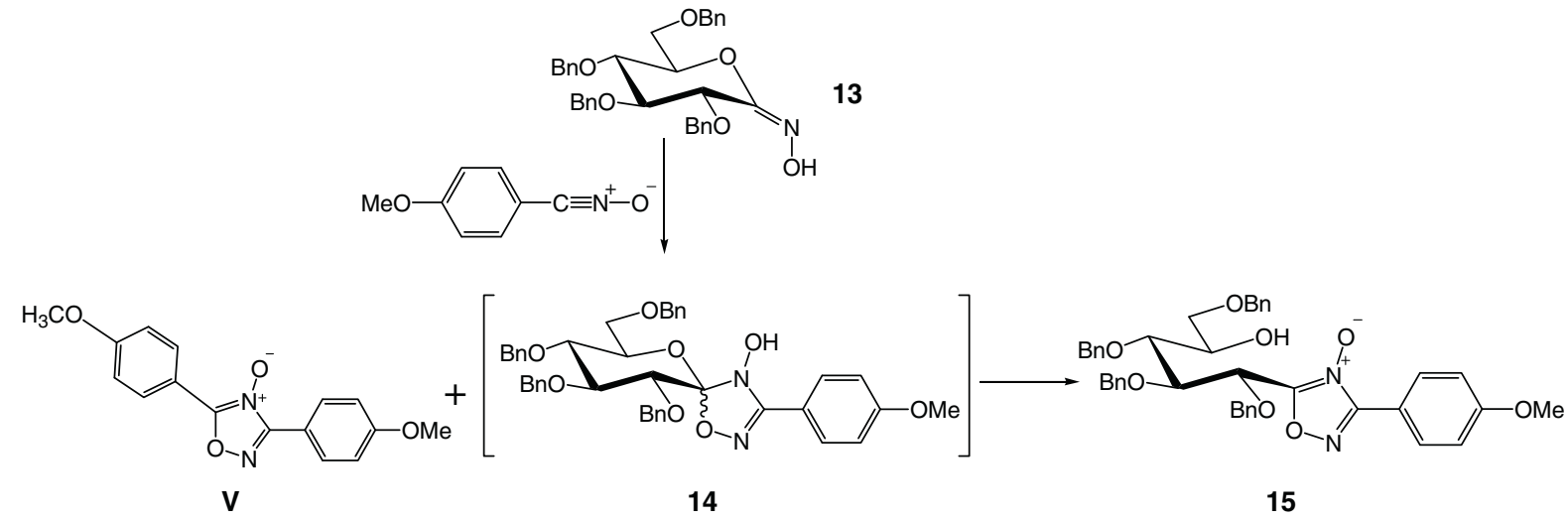

Scheme 3. Attempted cyclization of glucosyl-hydroximolactone $\mathbf{1 3 .}$

Table 3

Inhibition of rabbit muscle glycogen phosphorylase (RMGP) $b$

\begin{tabular}{llll}
\hline Compound & Inhibition $(\mu \mathrm{M})$ & Compound & Inhibition $(\mu \mathrm{M})$ \\
\hline $\mathbf{2 b}$ & IC50 $>10000$ & $(1 S)-\mathbf{4 a}$ & $K_{\mathrm{i}}=26$ \\
$\mathbf{2 c}$ & No inhibition at 625 & $(1 S)-\mathbf{4 b}$ & IC50 $=250$ \\
$\mathbf{2 e}$ & No inhibition at 312.5 & $(1 S)-\mathbf{4 c}$ & IC50 $=700$ \\
$\mathbf{2 g}$ & Insoluble in DMSO & $(1 S)-\mathbf{4 d}$ & $K_{\mathrm{i}}=48$ \\
$\mathbf{2 h}$ & Insoluble in DMSO & $(1 S)-\mathbf{4 e}$ & $K_{\mathrm{i}}=8.2$ \\
$\mathbf{2 i}$ & No inhibition at 625 & $(1 S)-\mathbf{4 g}$ & IC50 $=250$ \\
$\mathbf{9 b}$ & No inhibition at 625 & $(1 S)-\mathbf{4 h}$ & $K_{\mathrm{i}}=0.16$ \\
$\mathbf{9 c}$ & $K_{\mathrm{i}}=1800$ & $(1 R)-\mathbf{4 i}$ & No inhibition at 625 \\
\hline
\end{tabular}

Based on the binding peculiarities of these types of inhibitors as revealed by X-ray crystallography, we set up a new design principle towards better inhibitors. ${ }^{23}$ To validate this approach, we designed a synthesis of properly substituted glucopyranosylidene-spirooxathiazoles by NBS-mediated spiro-cyclization at the anomeric center of glucopyranosyl-hydroximothioate precursors. Attempted preparations of the corresponding aza-analogs, in which the sulphur atom is replaced by a nitrogen, failed and led to heteroaromatic structures with an acyclic carbohydrate moiety. The 2naphthyl substituted glucopyranosylidene-spiro-oxathiazole displayed the strongest inhibition with a $K_{\mathrm{i}}$ value of $160 \mathrm{nM}$. The present study provides the best inhibitor of GP in the glucose-based family and strengthens the need for additional investigations towards inhibition of this enzyme.

\section{Experimental}

\subsection{General methods}

Dichloromethane, chloroform and carbon tetrachloride were washed with water ( 3 times), then dried $\left(\mathrm{CaCl}_{2}\right)$ prior distillation over $\mathrm{CaH}_{2}$, and kept away from light. Triethylamine and water were distilled twice. Thin-layer chromatography (TLC) was carried out on aluminum sheets coated with Silica Gel $60 \mathrm{~F}_{254}$ (Merck). TLC plates were inspected by UV light $(\hat{\lambda}=254 \mathrm{~nm})$ and developed by treatment with a mixture of $10 \% \mathrm{H}_{2} \mathrm{SO}_{4}$ in $\mathrm{EtOH} / \mathrm{H}_{2} \mathrm{O}(1 / 1 \mathrm{v} / \mathrm{v})$ followed by heating. Brominated compounds were visualized on TLC plates by spraying first a $0.1 \% \mathrm{w} / \mathrm{v}$ fluorescein solution in absolute $\mathrm{MeOH}$, then a $1: 1$ mixture of $\mathrm{H}_{2} \mathrm{O}_{2}$ (30\% in water) and $\mathrm{AcOH}$. Upon gentle heating, bromo-compounds gave pink-colored spots. Silica gel col-

300 umn chromatography was performed with Geduran ${ }^{\circledR}$ Silica Gel Si $60(40-63 \mu \mathrm{m})$ purchased from Merck (Darmstadt, Germany). Preparative reversed phase chromatography (RP-18) was performed using a $15 \times 150 \mathrm{~mm}$ column of fully endcapped Silica Gel $100 \mathrm{C}_{18}$

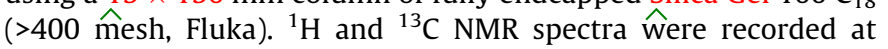

$23^{\circ} \mathrm{C}$ using Bruker DRX300 or DRX500 spectrometers with the residual solvent as the internal standard. The following abbreviations are used to explain the observed multiplicities: s, singlet; d, doublet; dd, doublet of doublet; ddd, doublet of doublet of doublet; t, triplet; td, triplet of doublet; q, quadruplet; m, multiplet; br, broad; p, pseudo. Structure elucidation was deduced from 1D and 2D NMR spectroscopy which allowed, in most cases, complete signal assignments based on COSY, HSQC, and HMBC correlations. Atom numbering follows that of the parent carbohydrate for the description of the sugar signals in the NMR spectra. Uncertain assignments are indicated by an asterisk. NMR solvents were purchased from Euriso-Top (Saint Aubin, France). HRMS (LSIMS) mass spectra were recorded in the positive mode using a Thermo Finnigan Mat 95 XL spectrometer. MS (ESI) mass spectra were recorded in the positive mode (unless stated otherwise) using a Thermo Finnigan LCQ spectrometer. Optical rotations were measured using a Perkin Elmer polarimeter. A collection of compounds $\mathbf{1}$ and $\mathbf{2}$ have been previously described except for their ${ }^{13} \mathrm{C}$ NMR data. ${ }^{32} 1$-Thio- $\beta$-D-glucopyranosyl hydroximothioates 2a,b,d,e were previously reported. ${ }^{32}$ Compound (1S)-3d was previously reported. ${ }^{22}$

\subsubsection{General procedure $A$ for the preparation}

of 0 -peracetylated hydroximothioates 1

Preparation adapted from previously reported procedures. ${ }^{21,32}$

$2,3,4,6$-Tetra- $O$-acetyl- 1 -thio- $\beta$-D-glucopyranose $(1 \mathrm{mmol}$ ) and hydroximoyl chloride ( $1.2 \mathrm{mmol}, 1.2$ equiv) were dissolved in $\mathrm{CH}_{2} \mathrm{Cl}_{2}(5 \mathrm{~mL})$. After adding triethylamine ( $3 \mathrm{mmol}, 3$ equiv), the mixture was stirred at room temperature and TLC monitoring showed completion of the reaction. The solvent was evaporated and the residue purified by flash silica gel column chromatography (PE/EtOAc 1:1, then 2:3) to afford the desired hydroximothioates 1.

\subsubsection{General procedure $B$ for the preparation of deacetylated compounds $2 \mathrm{i}$ and $(1 R)-4 \mathrm{i}$}

The acetylated compound $(0.25 \mathrm{mmol})$ was dissolved in a mixture of water $(5 \mathrm{~mL}), \mathrm{MeOH}(5 \mathrm{~mL})$ and triethylamine $(1 \mathrm{~mL})$. After stirring overnight at room temperature, TLC showed deacetylation was complete. Evaporation of the volatiles under reduced pressure afforded a residue corresponding to pure deacetylated product.

\subsubsection{General procedure $C$ for the preparation of spiro- oxathiazoles 3}

A solution of hydroximothioate $\mathbf{1}(1 \mathrm{mmol})$ and $\mathrm{N}$-bromosuccinimide ( $2 \mathrm{mmol})$ in $\mathrm{CCl}_{4}(20 \mathrm{~mL}$, for $\mathbf{3 b}-\mathbf{e}, \mathbf{g}, \mathbf{h})$ or $\mathrm{CHCl}_{3}(20 \mathrm{~mL}$ for 3a,f,i) was boiled and illuminated by a $60 \mathrm{~W}$ heat lamp for $45 \mathrm{~min}$. The reaction was diluted with EtOAc $(150 \mathrm{~mL})$ and the organic layer was washed with $5 \%$ aqueous $\mathrm{Na}_{2} \mathrm{SO}_{3}(2 \times 100 \mathrm{~mL})$ and water $(2 \times 100 \mathrm{~mL})$, dried $\left(\mathrm{Na}_{2} \mathrm{SO}_{4}\right)$, filtered and evaporated under 
350 reduced pressure. The residue was purified by flash column chromatography (PE then PE/EtOAc 65:35) to afford the desired acetylated spiro-oxathiazole 3.

\subsubsection{General procedure $D$ for the Zemplén deacetylation}

A solution of an acetylated compound 1 or $3(150 \mathrm{mg})$ and $\mathrm{NaOMe}(5 \mathrm{mg})$ in $\mathrm{MeOH}(15 \mathrm{~mL})$ was stirred at rt for $3 \mathrm{~h}$. The reaction was neutralized to $\mathrm{pH}$ 5-6 with Amberlite IR-120 resin $\left(\mathrm{H}^{+}\right.$ form) and the resin washed with $\mathrm{MeOH}(2 \times 10 \mathrm{~mL})$. The filtrate was evaporated under reduced pressure to afford the desired hydroximothioates $\mathbf{2}$ and spiro-oxathiazoles $\mathbf{4}$, respectively.

\section{$360 \quad$ 4.1.5. General procedure $E$ for the synthesis of $\boldsymbol{N}$-glycosyl- amidoximes 8}

Trimethyl phosphine ( $2.68 \mathrm{~mL}, 1 \mathrm{M}$ in toluene) was added with stirring to a solution of 2,3,4,6-tetra- $O$-acetyl- $\beta$-D-glucopyranosyl azide $7(1 \mathrm{~g}, 2.68 \mathrm{mmol})$ in dry dichloromethane $(10 \mathrm{~mL})$. After gas evolution had ceased and TLC showed complete transformation of the starting azide 7 (10-15 min), a hydroximoyl chloride ( 1 equiv) was added. After $20-30 \mathrm{~min}$, the reaction mixture was poured into water, and the organic layer was separated. The aqueous layer was extracted with dichloromethane $(2 \times 20 \mathrm{~mL})$. The organic layers were combined, washed with $10 \%$ aq sodium carbonate and water $(5 \times 20 \mathrm{~mL})$, dried $\left(\mathrm{Na}_{2} \mathrm{SO}_{4}\right)$, filtered and evaporated. The residue was purified by flash column chromatography (EtOAc/hexane, 1:3) or crystallization. An optimized synthesis of $\mathbf{8} \mathbf{c}$ is also presented below.

\subsection{Synthesis of 0 -peracetylated hydroximothioates 1}

4.2.1. 2,3,4,6-Tetra-O-acetyl-1-S-(Z)-4-nitrobenzhydroximoyl-1thio- $\beta$-D-glucopyranose $1 \mathrm{~b}$

From tetra- $O$-acetyl-1-thio- $\beta$-D-glucopyranose $(1.8 \mathrm{~g}, 5.3 \mathrm{mmol})$ according to general procedure A yielding $\mathbf{1 b}(1.38 \mathrm{~g}, 2.61 \mathrm{mmol}$, $53 \%)$ as a yellow crystalline product.

$R_{\mathrm{f}}=0.48($ PE/EtOAc, $1: 1) ;[\alpha]_{\mathrm{D}}^{25}+7.5($ c $0.57, \mathrm{MeOH})$, lit. $^{32}[\alpha]_{\mathrm{D}}$ +8.0 (c 1, $\left.\mathrm{CHCl}_{3}\right) ; \mathrm{mp}=156-157{ }^{\circ} \mathrm{C} ;{ }^{1} \mathrm{H} \mathrm{NMR}\left(360 \mathrm{MHz}, \mathrm{CDCl}_{3}\right) \delta$ $9.40(\mathrm{~s}, 1 \mathrm{H}, \mathrm{OH}), 8.30 \mathrm{~d}, 2 \mathrm{H}, J=7.9 \mathrm{~Hz}, \mathrm{H}-\mathrm{ar}), 7.62$ (d, $2 \mathrm{H}$, $J=7.9 \mathrm{~Hz}, \mathrm{H}-\mathrm{ar}), 5.11-5.02(\mathrm{~m}, 3 \mathrm{H}, \mathrm{H}-2 \mathrm{H}-3 \mathrm{H}-4), 4.63(\mathrm{~d}, 1 \mathrm{H}$, $J=9.2 \mathrm{~Hz}, \mathrm{H}-1$ ), 4.12 (dd, $1 \mathrm{H}, J=3.8$ and $11.9 \mathrm{~Hz}, \mathrm{H}-6), 4.01$ (dd, $\left.1 \mathrm{H}, J=2.6 \mathrm{~Hz}, \mathrm{H}-6^{\prime}\right), 3.24$ (ddd, $\left.1 \mathrm{H}, \mathrm{H}-5\right), 2.11,2.07$ (2s, $6 \mathrm{H}, \mathrm{CH}_{3} \mathrm{CO}$ ), $1.99\left(\mathrm{~s}, 6 \mathrm{H}, \mathrm{CH}_{3} \mathrm{CO}\right) ;{ }^{13} \mathrm{C} \mathrm{NMR}\left(90 \mathrm{MHz}, \mathrm{CDCl}_{3}\right) \delta 170.6,170.2(2 \mathrm{~s}$, $\left.\mathrm{CH}_{3} \mathrm{CO}\right), 169.3\left(\mathrm{~s}, 2 \mathrm{C}, \mathrm{CH}_{3} \mathrm{CO}\right), 149.4(\mathrm{C}=\mathrm{N}), 148.6,139.2(2 \mathrm{~s}, \mathrm{CH}-$ ar), 129.8 (s, 2C, CH-ar), 123.5 (s, 2C, CH-ar), 81.3 (C-1), 75.8, 73.4, 70.1, 67.8 (4s, C-2 C-3 C-4 C-5), 61.8 (C-6), 20.7, 20.6 (2s, 2C, $\mathrm{CH}_{3} \mathrm{CO}$ ), 20.4 (s, 2C, $\mathrm{CH}_{3} \mathrm{CO}$ ); Anal. Calcd for $\mathrm{C}_{21} \mathrm{H}_{24} \mathrm{~N}_{2} \mathrm{O}_{12} \mathrm{~S}$ (528.50): C, 47.73; H, 4.58; N, 5.30; S, 6.07. Found: C, 47.51; H, 4.76 ; N, 5.65; S, 5.99 .

\subsection{2. $2,3,4,6$-Tetra- 0 -acetyl-1- $S$ - $(Z)-4$-cyanobenzhydroximoyl-}

1-thio- $\beta$-D-glucopyranose $1 \mathrm{c}$

From tetra- $O$-acetyl-1-thio- $\beta$-D-glucopyranose $(1.5 \mathrm{~g}, 4.1 \mathrm{mmol})$ according to general procedure $\mathbf{A}$ yielding $\mathbf{1 c}(900 \mathrm{mg}, 1.77 \mathrm{mmol}$, $48 \%)$ as a white crystalline product.

$R_{\mathrm{f}}=0.19(\mathrm{PE} / \mathrm{EtOAc}, 2: 1) ;[\alpha]_{\mathrm{D}}^{25}+23($ c $0.28, \mathrm{MeOH}) ; \mathrm{mp}=165-$ $167{ }^{\circ} \mathrm{C} ;{ }^{1} \mathrm{H}$ NMR $\left(360 \mathrm{MHz}, \mathrm{CDCl}_{3}\right) \delta 10.10(\mathrm{~s}, 1 \mathrm{H}, \mathrm{OH}), \hat{7} .80-7.70$ (m, 4H, H-ar), 5.10-5.00 (m, 3H, H-2 H-3 H-4), 4.6仓 (d, $1 \mathrm{H}$, $J=9.2 \mathrm{~Hz}, \mathrm{H}-1), \hat{4} .12(\mathrm{dd}, 1 \mathrm{H}, J=3.8$ and $11.9 \mathrm{~Hz}, \mathrm{H}-6), 3.98$ (dd, $\left.1 \mathrm{H}, J=2.6 \mathrm{~Hz}, \mathrm{H}-6^{\prime}\right), 3.22$ (ddd, $\left.1 \mathrm{H}, \mathrm{H}-5\right), 2.09,2.05\left(2 \mathrm{~s}, 6 \mathrm{H}, \mathrm{CH}_{3} \mathrm{CO}\right.$ ), 1.99 (s, 6H, $\left.\mathrm{CH}_{3} \mathrm{CO}\right) ;{ }^{13} \mathrm{C} \mathrm{NMR}\left(90 \mathrm{MHz}, \mathrm{CDCl}_{3}\right) \delta 170.6,170.1,(2 \mathrm{~s}$, $\left.\mathrm{CH}_{3} \mathrm{CO}\right), 169.3\left(\mathrm{~s}, 2 \mathrm{C}, \mathrm{CH}_{3} \mathrm{CO}\right), 149.0(\mathrm{C}=\mathrm{N}), 137.5,132.1$ (2s, CHar), 129.4 (s, 2C, CH-ar), 117.9 (s, 2C, CH-ar), $113.4(\mathrm{CN}), 81.1$ (C1), 75.6, 73.4, 70.0, 67.7 (4s, C-2 C-3 C-4 C5), 61.7 (C-6), 20.6, 20.4 (2s, $\mathrm{CH}_{3} \mathrm{CO}$ ), 20.3 (s, 2C, $\mathrm{CH}_{3} \mathrm{CO}$ ); Anal. Calcd for $\mathrm{C}_{21} \mathrm{H}_{24} \mathrm{~N}_{2} \mathrm{O}_{10} \mathrm{~S}$ (508.11): C, 51.96; H, 4.76; N, 5.51; S, 6.31. Found: C, 51.71; H, $4.88 ; \mathrm{N}, 5.32 ; \mathrm{S}, 6.11$.
4.2.3. 2,3,4,6-Tetra-O-acetyl-1-S-(Z)-4-methoxybenzhydroximoyl1-thio- $\beta$-D-glucopyranose $1 \mathrm{e}$

From tetra-O-acetyl-1-thio- $\beta$-D-glucopyranose (1.63 g, $4.47 \mathrm{mmol})$ according to general procedure A yielding 1 e $(1.17 \mathrm{~g}, 2.28 \mathrm{mmol}$, $51 \%)$ as a white crystalline product.

$R_{\mathrm{f}}=0.27(\mathrm{PE} /$ EtOAc, $1: 1) ;[\alpha]_{\mathrm{D}}^{25}+29\left(c 0.44, \mathrm{CH}_{2} \mathrm{Cl}_{2}\right) ;$ lit. $^{32}[\alpha]_{\mathrm{D}}$ +13 (c 1, $\mathrm{CHCl}_{3}$ ); $\mathrm{mp}=157-161^{\circ} \mathrm{C}$ lit. $^{32} \mathrm{mp} \equiv 150{ }^{\circ} \mathrm{C} ;{ }^{1} \mathrm{H}$ NMR

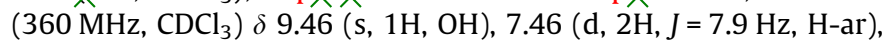
$6.93(\mathrm{~d}, 2 \mathrm{H}, J=7.9 \mathrm{~Hz}, \mathrm{H}-\mathrm{ar}), 5.10-4.99(\mathrm{~m}, 3 \mathrm{H}, \mathrm{H}-2 \mathrm{H}-3 \mathrm{H}-4)$, $4.50(\mathrm{~d}, 1 \mathrm{H}, J=10.5 \mathrm{~Hz}, \mathrm{H}-1), 4.12(\mathrm{dd}, 1 \mathrm{H}, J=3.6$ and $11.9 \mathrm{~Hz}, \mathrm{H}-$ 6), $3.98\left(\mathrm{dd}, 1 \mathrm{H}, J=1.2 \mathrm{~Hz}, \mathrm{H}-6^{\prime}\right), 3.86\left(\mathrm{~s}, 3 \mathrm{H}, \mathrm{OCH}_{3}\right), 3.13$ (ddd, $1 \mathrm{H}, \mathrm{H}-5), 2.10,2.04\left(2 \mathrm{~s}, 6 \mathrm{H}, \mathrm{CH}_{3} \mathrm{CO}\right), 1.96\left(\mathrm{~s}, 6 \mathrm{H}, \mathrm{CH}_{3} \mathrm{CO}\right) ;{ }^{13} \mathrm{C}$ NMR (90 MHz, $\left.\mathrm{CDCl}_{3}\right) \delta 170.6,170.2$ (2s, $\left.\mathrm{CH}_{3} \mathrm{CO}\right), 169.2$ (s, 2C, $\left.\mathrm{CH}_{3} \mathrm{CO}\right), 160.8(\mathrm{C}=\mathrm{N}), 152.2,130.3,124.5(2 \mathrm{~s}, \mathrm{CH}-\mathrm{ar}), 113.7(2 \mathrm{~s}$, CH-ar), 81.4 (C-1), 75.3, 73.7, 69.4, 67.8 (4s, C-2 C-3 C-4 C5), 61.8 (C-6), $55.3\left(\mathrm{OCH}_{3}\right), 20.6,20.5,20.4,20.3\left(4 \mathrm{~s}, \mathrm{CH}_{3} \mathrm{CO}\right)$; Anal. C्रalcd for $\mathrm{C}_{22} \mathrm{H}_{27} \mathrm{NO}_{11} \mathrm{~S}$ (513.52): C, 51.46; H, 5.30; N, 5.51; S, 6.24. Found: C, 51.62; H, 5.46; N, 5.50; S, 6.01.

4.2.4. 2,3,4,6-Tetra-O-acetyl-1-S-(Z)-3,4,5-trimethoxybenzhydroximoyl-1-thio- $\beta$-D-glucopyranose if

Tetra- $O$-acetyl-1-thio- $\beta$-D-glucopyranose $(553 \mathrm{mg}, 1.57 \mathrm{mmol}$ ) dissolved in $\mathrm{CH}_{2} \mathrm{Cl}_{2}(30 \mathrm{~mL})$ was reacted with 3,4,5-trimethoxybenzhydroximoyl chloride ( $4.92 \mathrm{mmol}, 3$ e्रquiv) upon stirring at room temperature in the presence of $\mathrm{NEt}_{3}(3 \mathrm{~mL}$, $22 \mathrm{mmol}, 14$ equiv) and under argon. After the reaction was over ( $\sim 30 \mathrm{~min}$ ), aqueous washings and concentration afforded a crude oil which was purified by column chromatography (PE/EtOAc $1: 1$, then $2: 3)$, yielding $\mathbf{1 f}(770 \mathrm{mg}, 1.35 \mathrm{mmol}, 85 \%$ yield) as a colorless foam.

$R_{\mathrm{f}}=0.23($ PE/EtOAc, $1: 1) ;[\alpha]_{\mathrm{D}}^{20}+27.8\left(\mathrm{c} 1, \mathrm{CH}_{2} \mathrm{Cl}_{2}\right) ;{ }^{1} \mathrm{H} \mathrm{NMR}$ $\left(300 \mathrm{MHz}, \mathrm{CDCl}_{3}\right) \delta 9.12(\mathrm{~s}, 1 \mathrm{H}, \mathrm{OH}), 6.75(\mathrm{~s}, 2 \mathrm{H}, \mathrm{H}-\mathrm{ar}), 5.03(\mathrm{~m}$, $3 \mathrm{H}, \mathrm{H}-2 \mathrm{H}-3 \mathrm{H}-4), 4.68$ (d, $1 \mathrm{H}, J=9.5 \mathrm{~Hz}, \mathrm{H}-1), 4.06$ (dd, $1 \mathrm{H}$, $J=3.8$ and $12.5 \mathrm{~Hz}, \mathrm{H}-6), 3.93$ (dd, $1 \mathrm{H}, J=2.3 \mathrm{~Hz}, \mathrm{H}-6^{\prime}$ ), 3.84 (s, $9 \mathrm{H}, \mathrm{OMe}), 3.19$ (ddd, $1 \mathrm{H}, \mathrm{H}-5), 2.00,1.99,1.94,1.92(4 \mathrm{~s}, 12 \mathrm{H}$, $\left.\mathrm{CH}_{3} \mathrm{CO}\right) ;{ }^{13} \mathrm{C}$ NMR $\left(75 \mathrm{MHz}, \mathrm{CDCl}_{3}\right) \delta 171.1,170.7,170.2,169.7$ (4s, $\left.\mathrm{CH}_{3} \mathrm{CO}\right), 153.6(\mathrm{~s}, 2 \mathrm{C}), 151.8(\mathrm{C}=\mathrm{N}), 140.0,128.4,106.8(\mathrm{~s}, 2 \mathrm{C}$, CH-ar), 82.1 (C-1), 76.2 (C-5), 74.1, 70.8, 68.1 (3s, C-2 C-3 C-4), 61.8 (C-6), 21.0, 20.92, 20.91, 20.8 (4s, $\left.\mathrm{CH}_{3} \mathrm{CO}\right)$; Anal. Calcd for $\mathrm{C}_{24} \mathrm{H}_{31} \mathrm{NO}_{13} \mathrm{~S}$ (573.57): H, 5.45; N, 2.44; O, 36.26. Found: $\mathrm{H}, 5.20$; $\mathrm{N}, 2.25 ; 0,35.46$

4.2.5. 2,3,4,6-Tetra-O-acetyl-1-S-(Z)-(4-phenyl)-benzhydroximoyl-1-thio- $\beta$-D-glucopyranose $1 \mathrm{~g}$

From tetra-O-acetyl-1-thio- $\beta$-D-glucopyranose ( $1.94 \mathrm{~g}, 5.32 \mathrm{mmol}$ ) according to general procedure A yielding $1 \mathrm{~g}(1.20 \mathrm{~g}, 2.14 \mathrm{mmol}$, $40 \%)$ as a white crystalline product.

$R_{\mathrm{f}}=0.28($ PE/EtOAc, $1: 1) ;[\alpha]_{\mathrm{D}}^{25}+5.7($ c 0.17, MeOH); $\mathrm{mp}=101-$ $103{ }^{\circ} \mathrm{C} ;{ }^{1} \mathrm{H}$ NMR $\left(360 \mathrm{MHz}, \mathrm{CDCl}_{3}\right) \delta 9.53(\mathrm{~s}, 1 \mathrm{H}, \mathrm{OH}), \hat{7} .72 \widehat{-7} .63$ (m, 6H, H-ar), 7.55-7.41 (m, 3H, H-ar), 5.16-5.00 (m, 3̂े, H-2 H$3 \mathrm{H}-4), 4.55$ (d, $1 \mathrm{H}, J=9.2 \mathrm{~Hz}, \mathrm{H}-1), \hat{4.25}(\mathrm{dd}, 1 \mathrm{H}, J=3.6$ and $11.9 \mathrm{~Hz}, \mathrm{H}-6$ ), 4.15 (dd, $1 \mathrm{H}, J=2.6 \mathrm{~Hz}, \mathrm{H}-6^{\prime}$ ), 3.15 (ddd, $1 \mathrm{H}, \mathrm{H}-5$ ), 2.12, 2.09, 2.01, $1.98\left(4 \mathrm{~s}, 12 \mathrm{H}, \mathrm{CH}_{3} \mathrm{CO}\right) ;{ }^{13} \mathrm{C} \mathrm{NMR}\left(90 \mathrm{MHz}, \mathrm{CDCl}_{3}\right)$ $\delta$ 170.6, 170.2, $169.2\left(4 \mathrm{~s}, \mathrm{CH}_{3} \mathrm{CO}\right), 151.9(\mathrm{C}=\mathrm{N}), 142.7,139.8$, 131.9, 129.3, 128.9 (s, 2C), 127.8, 127.6, 127.3, 127.0 (s, 2C), 126.9 (CH-ar), 81.3 (C-1), 75.8, 73.7, 69.9, 67.8 (4s, C-2 C-3 C-4 C-5), 61.2 (C-6), 20.6, 20.5, 20.4, 20.3 (4s, $\mathrm{CH}_{3} \mathrm{CO}$ ); Anal. Calcd for $\mathrm{C}_{27} \mathrm{H}_{29} \mathrm{NO}_{10} \mathrm{~S}$ (559.60): C, 57.95; H, 5.22; N, 2.50; S, 5.73. Found: C, 57.71; H, 4.99; N, 2.66; S, 5.52.

4.2.6. 2,3,4,6-Tetra-O-acetyl-1-S-(Z)-2-naphthoyl-hydroximoyl1-thio- $\beta$-D-glucopyranose $1 \mathrm{~h}^{23}$

From tetra-O-acetyl-1-thio- $\beta$-D-glucopyranose $\quad(875 \mathrm{mg}$, $2.40 \mathrm{mmol}$ ) according to general procedure A yielding $\mathbf{1 h}$ (999 $\mathrm{mg}, 1.87 \mathrm{mmol}, 78 \%$ ) as a white crystalline product. 
4.2.7. 2,3,4,6-Tetra-O-acetyl-1-S-(Z)-isonicotinoyl-hydroximoyl1-thio- $\beta$-D-glucopyranose $1 \mathrm{i}$

2,3,4,6-Tetra-O-acetyl-1-thio- $\beta$-D-glucopyranose (107 mg, 0.29 $\mathrm{mmol}$ ) and 4-pyridyl-hydroximoyl chloride (250 $\mathrm{mg}, 1.59 \mathrm{mmol}$, 5.6 equiv) were introduced in a $25 \mathrm{~mL}$ flask. After the flask was flushed with argon, anhydrous dichloromethane $(6 \mathrm{~mL})$ and triethylamine ( $0.53 \mathrm{~mL}, 3.77 \mathrm{mmol}, 13$ equiv) were added with stirring. After the dark brown solution was stirred for $30 \mathrm{~min}$ at room temperature, the reaction was finished (TLC). The mixture, diluted with dichloromethane $(25 \mathrm{~mL})$, was washed with a satd aq $\mathrm{NaHCO}_{3}$ solution $(25 \mathrm{~mL})$, then with water $(25 \mathrm{~mL})$. The organic layer was dried $\left(\mathrm{MgSO}_{4}\right)$ and concentrated under reduced pressure to afford a crude yellow oil (245 mg) which was subjected to column chromatography (PE/EtOAc 2:3 gradually changed to $0: 1$, with $0.1 \%$ $\mathrm{Et}_{3} \mathrm{~N}$ ) to afford an orange-colored solid. After treatment of a dichloromethane solution with charcoal, $\mathbf{1 i}$ was obtained a white solid upon filtration and evaporation (112 mg, $0.23 \mathrm{mmol}, 78 \%$ yield).

$R_{\mathrm{f}}=0.12(\mathrm{PE} / \mathrm{EtOAc}, 2: 3) ;[\alpha]_{\mathrm{D}}^{20}+9.3\left(\right.$ c $\left.1, \mathrm{CH}_{2} \mathrm{Cl}_{2}\right)$; $\mathrm{Mp}=139-$ $140{ }^{\circ} \mathrm{C} ;{ }^{1} \mathrm{H}$ NMR $\left(300 \mathrm{MHz}, \mathrm{CD}_{3} \mathrm{COCD}_{3}\right) \delta 11.61(\mathrm{~s}, 1 \mathrm{H}, \mathrm{OH}), 8.69$ (dd, $2 \mathrm{H}, J=1.6$ and $4.4 \mathrm{~Hz}, \mathrm{H}-\mathrm{ar}$ ), 7.56 (dd, 2H, H-ar), 5.23 (t, 1H, $J=9.0 \mathrm{~Hz} \mathrm{H}-3), 5.01(\mathrm{t}, 1 \mathrm{H}, \mathrm{H}-2), 4.98(\mathrm{t}, 1 \mathrm{H}, \mathrm{H}-4), 4.95(\mathrm{~d}, 1 \mathrm{H}$, $J=10.0 \mathrm{~Hz}, \mathrm{H}-1$ ), 4.12 (dd, $1 \mathrm{H}, J=5.5$ and $12.5 \mathrm{~Hz}, \mathrm{H}-6$ ), 3.98 (dd, $\left.1 \mathrm{H}, J=2.3 \mathrm{~Hz}, \mathrm{H}-6^{\prime}\right), 3.56$ (ddd, $\left.1 \mathrm{H}, \mathrm{H}-5\right), 2.06,2.02,1.94,1.92$ (4s, $\left.12 \mathrm{H}, \mathrm{CH}_{3} \mathrm{CO}\right) ;{ }^{13} \mathrm{C} \mathrm{NMR}\left(75 \mathrm{MHz}, \mathrm{CD}_{3} \mathrm{COCD}_{3}\right) \delta 171.6,171.1$, 170.9, 170.8 (4s, $\left.\mathrm{CH}_{3} \mathrm{CO}\right), 152.0$ (s, 2C, $\left.\mathrm{CH}-\mathrm{ar}\right), 149.9(\mathrm{C}=\mathrm{N}), 143.0$, 124.9 (s, 2C, CH-ar), 82.5 (C-1), 77.3 (C-5), 75.0 (C-3), 72.1, 70.0 (2s, C-2 C-4), 63.8 (C-6), 21.6, 21.5, 21.4, 21.3 (4s, $\left.\mathrm{CH}_{3} \mathrm{CO}\right)$; HRMS $(\mathrm{FAB}>0) \mathrm{C}_{20} \mathrm{H}_{25} \mathrm{~N}_{2} \mathrm{O}_{10} \mathrm{~S}[\mathrm{M}+\mathrm{H}]^{+}$Calcd 485.1230, found 485.1233; Anal. Calcd for $\mathrm{C}_{20} \mathrm{H}_{24} \mathrm{~N}_{2} \mathrm{O}_{10} \mathrm{~S}(484.28)$ : C, 49.58; $\mathrm{H}, 4.99 ; \mathrm{N}, 5.78$; O, 33.02; S, 6.62. Found: C, 49.51; H, 4.96; N, 5.70; O, 32.95.

4.2.8. 2,3,4,6-Tetra-O-acetyl-1-S-(Z)-isonicotinoyl-hydroximoyl1-thio- $\boldsymbol{\beta}$-D-glucopyranose- $\mathbf{N}$-oxide $\mathbf{1 j}$

A solution of $1 \mathbf{i}(195 \mathrm{mg}, 0.4 \mathrm{mmol})$ in chloroform $(10 \mathrm{~mL})$ was cooled down to $0{ }^{\circ} \mathrm{C}$ before the addition of $\mathrm{m}$-chloroperbenzoic acid (139 mg, $0.8 \mathrm{mmol}, 2$ equiv). After $8 \mathrm{~h}$, TLC monitoring showed two main polar products. After concentration under reduced pressure, the crude mixture was purified by flash column chromatography (EtOAc containing $0.1 \% \mathrm{Et}_{3} \mathrm{~N}$ gradually enriched in $\mathrm{MeOH}$ from $0 \%$ to $6 \%$ ) affording $\mathbf{1 j}$ (62 $\mathrm{mg}, 0.12 \mathrm{mmol}, 30 \%$ yield) as an orange solid. When the reaction was carried out with a lower amount of $m$-CPBA ( 1 equiv), the yield was $25 \%$.

$R_{\mathrm{f}}=0.20(\mathrm{EtOAc} / \mathrm{MeOH}, 9: 1) ;[\alpha]_{\mathrm{D}}^{20}-17.7$ ( c 0.53, MeOH); $\mathrm{mp}=$ $152{ }^{\circ} \mathrm{C}$ darkening, $182{ }^{\circ} \mathrm{C}$ decomposition; ${ }^{1} \mathrm{H}$ NMR $(300 \mathrm{MHz}$, $\left.\mathrm{CD}_{3} \mathrm{COCD}_{3}\right) \delta 11.49(\mathrm{~s}, 1 \mathrm{H}, \mathrm{OH}), 8.07$ (d, $\left.2 \mathrm{H}, J=7.2 \mathrm{~Hz}, \mathrm{H}-\mathrm{ar}\right), 7.50$ (d, $2 \mathrm{H}, \mathrm{H}-\mathrm{ar}), 5.14$ (t, $1 \mathrm{H}, J=9.2 \mathrm{~Hz}, \mathrm{H}-3), 4.98$ (d, $1 \mathrm{H}, J=10.1 \mathrm{~Hz}$, $\mathrm{H}-1$ ), 4.89 (dd, $1 \mathrm{H}, J=9.0 \mathrm{~Hz}, \mathrm{H}-2), 4.88(\mathrm{t}, 1 \mathrm{H}, J=9.7 \mathrm{~Hz}, \mathrm{H}-4)$, $4.04(\mathrm{dd}, 1 \mathrm{H}, J=5.5$ and $12.4 \mathrm{~Hz}, \mathrm{H}-6), 3.93(\mathrm{dd}, 1 \mathrm{H}, J=2.3 \mathrm{~Hz}, \mathrm{H}-$ $\left.6^{\prime}\right), 3.68$ (ddd, $\left.1 \mathrm{H}, \mathrm{H}-5\right), 1.92,1.89,1.83,1.80\left(4 \mathrm{~s}, 12 \mathrm{H}, \mathrm{CH}_{3} \mathrm{CO}\right)$; ${ }^{13} \mathrm{C}$ NMR $\left(75 \mathrm{MHz}, \mathrm{CD}_{3} \mathrm{COCD}_{3}\right)$ d $170.2,169.7,169.4,169.3(4 \mathrm{~s}$, $\mathrm{CH}_{3} \mathrm{CO}$ ), $147.2(\mathrm{C}=\mathrm{N}), 139.3$ (s, 2C, $\left.\mathrm{CH}-\mathrm{ar}\right),\left(\mathrm{C}^{\mathrm{IV}}\right.$ not seen), 126.3 (s, 2C, CH-ar), 81.3 (C-1), 75.7 (C-5), 73.5 (C-3), 70.6 (C-2), 68.5 (C-4), 62.5 (C-6), 20.0 (m, 4C, $\left.\mathrm{CH}_{3} \mathrm{CO}\right)$.

\subsection{Deacetylation of $\boldsymbol{O}$-peracetylated hydroximothioates}

\subsubsection{1-S-(Z)-4-Nitrobenzhydroximoyl-1-thio- $\beta$-D-glucopyranose} $2 b$

From compound $\mathbf{1 b}(200 \mathrm{mg}, 0.38 \mathrm{mmol}$ ) according to general procedure D yielding $\mathbf{2 b}$ ( $111 \mathrm{mg}, 0.3 \mathrm{mmol}, 82 \%$ ) as a white crystalline product.

$R_{\mathrm{f}}=0.12\left(\mathrm{CHCl}_{3} / \mathrm{MeOH}, 9: 1\right) ;[\alpha]_{\mathrm{D}}^{25}+7.5(c$ 0.56, MeOH$)$, lit. $^{32}[\alpha]_{\mathrm{D}}^{25}$ $+5(c 1, \mathrm{MeOH}) ; \mathrm{mp}=184-187{ }^{\circ} \mathrm{C} ;{ }^{1} \mathrm{H} \mathrm{NMR}\left(360 \mathrm{MHz}, \mathrm{D}_{2} \mathrm{O}\right) \delta 8.27(\mathrm{~d}$, $2 \mathrm{H}, J=7.9 \mathrm{~Hz}, \widehat{\mathrm{H}}$-ar), $7.72(\mathrm{~d}, 2 \mathrm{H}, J=7.9 \mathrm{~Hz}, \mathrm{H}-\mathrm{ar}), 4.24(\mathrm{~d}, 1 \mathrm{H}$, $J=9.2 \mathrm{~Hz}, \mathrm{H}-1), 3.58$ (dd, $1 \mathrm{H}, J=1.2$ and $11.9 \mathrm{~Hz}, \mathrm{H}-6), 3.51(\mathrm{dd}, 1 \mathrm{H}$, $\left.\hat{J}=3.9 \mathrm{~Hz}, \mathrm{H}-6^{\prime}\right), 3.36,3.28,3.20(3 \mathrm{t}, 3 \mathrm{H}, J=9.2 \mathrm{~Hz}, \mathrm{H}-2 \mathrm{H}-3 \mathrm{H}-4)$,
2.66 (ddd, $1 \mathrm{H}, \mathrm{H}-5) ;{ }^{13} \mathrm{C}$ NMR (90 MHz, $\left.\mathrm{D}_{2} \mathrm{O}\right) \delta 154.5(\mathrm{C}=\mathrm{N}), 149.9$, 140.1, 131.6 (s, 2C, CH-ar), 125.0 (s, 2C, CH-ar), 84.4 (C-1), 81.5, 78.5, 73.6, 70.3 (4s, C-2 C-3 C-4 C-5), 61.7 (C-6); Anal. Calcd for $\mathrm{C}_{13} \mathrm{H}_{16} \mathrm{~N}_{2} \mathrm{O}_{8} \mathrm{~S}$ (360.34): C., 43.33; H, 4.48; N, 7.77; S, 8.90. Found: C, $43.44 ; \mathrm{H}, 4.26$; N, 7.16; S, 8.70 .

\subsubsection{1-S-(Z)-4-Cyanobenzhydroximoyl-1-thio- $\beta$-D-glucopyranose $2 c$}

From compound 1c (200 mg, $0.39 \mathrm{mmol})$ according to general procedure D yielding (122 $\mathrm{mg}, 0.36 \mathrm{mmol}, 86 \%)$ as a slightly colored crystalline product.

$R_{\mathrm{f}}=0.74 \quad\left(\mathrm{CHCl}_{3} / \mathrm{MeOH}, \quad 7: 3\right) ;[\alpha]_{\mathrm{D}}^{20}+23 \quad$ (c $\left.0.27, \mathrm{MeOH}\right)$; $\mathrm{mp}=156-160{ }^{\circ} \mathrm{C} ;{ }^{1} \mathrm{H} \quad \mathrm{NMR} \quad\left(360 \mathrm{MHz}, \mathrm{D}_{2} \mathrm{O}\right) \quad \delta \quad 7.80 \quad(\mathrm{~d}, \quad 2 \mathrm{H}$, $J=7.9 \mathrm{~Hz}, \mathrm{H}-\mathrm{ar}), 7.72$ (d, $2 \mathrm{H}, J=7.9 \mathrm{~Hz}, \mathrm{H}-\mathrm{ar}), 4.24(\mathrm{~d}, 1 \mathrm{H}$, $J=9.2 \mathrm{~Hz}, \mathrm{H}-1), 3.62(\mathrm{dd}, 1 \mathrm{H}, J=5.3$ and $13.2 \mathrm{~Hz}, \mathrm{H}-6), 3.52$ (dd, $\left.1 \mathrm{H}, J=1.3 \mathrm{~Hz}, \mathrm{H}-6^{\prime}\right), 3.40-3.10$ (m, 3H, H-2 H-3 H-4), 2.75 (ddd, $1 \mathrm{H}, \mathrm{H}-5) ;{ }^{13} \mathrm{C}$ NMR (90 MHz, $\left.\mathrm{D}_{2} \mathrm{O}\right) \delta 148.3(\mathrm{C}=\mathrm{N}) 139.7,132.8$ (2s, CH-ar), 132.6 (2s, CH-ar), 119.5, $111.0(\mathrm{CN}), 83.3(\mathrm{C}-1), 80.0$, 77.2, 72.6, 69.2 (4s, C-2 C-3 C-4 C-5), 60.4 (C-6); Anal. Calcd for $\mathrm{C}_{14} \mathrm{H}_{16} \mathrm{~N}_{2} \mathrm{O}_{6} \mathrm{~S}$ (340.36): C, 49.41; H, 4.74; N, 8.23; S, 9.42. Found: C, 49.24; H, 4.66; N, 8.42; S, 9.12.

\subsubsection{1-S-(Z)-4-Methoxybenzhydroximoyl-1-thio- $\beta$-D-glucopyr-} anose $2 \mathrm{e}$

From compound 1e (300 mg, $0.58 \mathrm{mmol}$ ) according to general procedure D yielding $\mathbf{2 e}$ (172 $\mathrm{mg}, 0.50 \mathrm{mmol}, 86 \%)$ as a white crystalline product.

$R_{\mathrm{f}}=0.70\left(\mathrm{CHCl}_{3} / \mathrm{MeOH}, 7: 3\right) ;[\alpha]_{\mathrm{D}}^{25}-85(c$ 0.31, MeOH$) \operatorname{lit}^{32}[\alpha]_{\mathrm{D}}^{25}$ -7 (c 1, MeOH); mp $=146-148{ }^{\circ} \mathrm{C} ;{ }^{1} \mathrm{H}$ NMR $\left(360 \mathrm{MHz}, \mathrm{D}_{2} \mathrm{O}\right) \delta 7.45$ (d, $2 \mathrm{H}, J=7.9 \mathrm{~Hz}, \mathrm{H}-\mathrm{ar}), 7.04$ (d, $2 \mathrm{H}, J=7.9 \mathrm{~Hz}, \mathrm{H}-\mathrm{ar}), 4.22$ (d, $1 \mathrm{H}$, $J=9.2 \mathrm{~Hz}, \mathrm{H}-1$ ), 3.85 (s, 3H, OCH $)_{3}, 3.20-3.44$ (m, 3H, H-2 H-3 H4), $3.68(\mathrm{dd}, 1 \mathrm{H}, J=3.9$ and $13.2 \mathrm{~Hz}, \mathrm{H}-6), 3.56(\mathrm{dd}, 1 \mathrm{H}, J=2.6 \mathrm{~Hz}$, $\left.\mathrm{H}-6^{\prime}\right), 2.75$ (ddd, 1H, H-5); ${ }^{13} \mathrm{C}$ NMR $\left(90 \mathrm{MHz}, \mathrm{D}_{2} \mathrm{O}\right) \delta 159.5$ $(\mathrm{C}=\mathrm{N}), 151.0,130.9$ (2s, CH-ar), 126.7 (2s, CH-ar), 113.8, 83.2 (C1), 79.9, 77.3, 72.3, 69.1 (4s, C-2 C-3 C-4 C-5), 60.4 (C-6), 55.6 $\left(\mathrm{OCH}_{3}\right)$; Anal. Calcd for $\mathrm{C}_{14} \mathrm{H}_{19} \mathrm{NO}_{7} \mathrm{~S}$ (345.37): $\mathrm{C}, 48.69 ; \mathrm{H}, 5.55 ; \mathrm{N}$, 4.06; S, 9.28. Found: C, 48.44; H, 5.36; N, 4.26; S, 9.02 .

\subsubsection{1-S-(Z)-(4-Phenyl)-benzhydroximoyl-1-thio- $\beta$-D-glucopyr-} anose $2 \mathrm{~g}$

From compound $1 \mathrm{~g}$ ( $300 \mathrm{mg}, 0.53 \mathrm{mmol}$ ) according to general procedure D yielding (190 mg, $0.48 \mathrm{mmol}, 90 \%$ ) as a yellow oil.

$R_{\mathrm{f}}=0.58\left(\mathrm{CHCl}_{3} / \mathrm{MeOH}, 7: 3\right) ;[\alpha]_{\mathrm{D}}^{25}+5.7($ c $0.17, \mathrm{MeOH}) ;{ }^{1} \mathrm{H} \mathrm{NMR}$ $\left(360 \mathrm{MHz}, \mathrm{DMSO}-d_{6}\right) \delta 8.40(\mathrm{~s}, 1 \mathrm{H}, \mathrm{OH}), 7.52-7.20(\mathrm{~m}, 9 \mathrm{H}, \mathrm{H}-\mathrm{ar})$, $4.98-4.51(\mathrm{~m}, 4 \mathrm{H}, \mathrm{OH}), 4.15(\mathrm{~d}, 1 \mathrm{H}, J=9.2 \mathrm{~Hz}, \mathrm{H}-1), 4.24(\mathrm{dd}, 1 \mathrm{H}$, $J=5.8$ and $13.2 \mathrm{~Hz}, \mathrm{H}-6), 4.17$ (dd, $\left.1 \hat{\mathrm{H},} J=2.6 \mathrm{~Hz}, \mathrm{H}-6^{\prime}\right) 4.16-3.72$ (m, 3H, H-2 H-3 H-4), 3.24 (ddd, $1 \mathrm{H}, \mathrm{H}-5) ;{ }^{13} \mathrm{C}$ NMR $(90 \mathrm{MHz}$, DMSO- $\left.d_{6}\right) \delta 151.1(\mathrm{C}=\mathrm{N}), 140.9,140.1,129.9,129.7,129.6,129.5$, 129.3, 128.2, 128.1, 12个.1, 126.9, 126.7, 84.4 (C-1), 80.4, 79.5, 73.8, 70.2 (4s, C-2 C-3 C-4 C-5), 60.9 (C-6); Anal. Calcd for $\mathrm{C}_{19} \mathrm{H}_{21} \mathrm{NO}_{6} \mathrm{~S}$ (391.45): C, 58.30; H, 5.41; N, 3.58; S, 8.19. Found: C, 58.14; H, 5.16; N, 3.12; S, 8.02.

\subsubsection{1-S-(Z)-2-Naphthoyl-hydroximoyl-1-thio- $\beta$-D-glucopyr- anose $2 \mathrm{~h}$}

From compound $\mathbf{1 h}$ (343 $\mathrm{mg}, 0.64 \mathrm{mmol}$ ) according to general procedure D yielding ( $200 \mathrm{mg}, 0.55 \mathrm{mmol}, 85 \%$ ) as a yellow oil.

$R_{\mathrm{f}}=0.68\left(\mathrm{CHCl}_{3} / \mathrm{MeOH}, 7: 3\right) ;[\alpha]_{\mathrm{D}}^{25}+4.4($ c $0.3, \mathrm{MeOH}) ; \mathrm{mp}=150-$ $152{ }^{\circ} \mathrm{C} ;{ }^{1} \mathrm{H}$ NMR $\left(360 \mathrm{MHz}, \mathrm{D}_{2} \mathrm{O}\right) \delta 8.04-7.54$ (m, 7H, H-ar), $4 . \hat{24}(\mathrm{~d}$, $1 \mathrm{H}, J=10.6 \mathrm{~Hz}, \mathrm{H}-1$ ), 3.56 (dd, $1 \hat{\mathrm{H}}, J=3.9$ and $13.2 \mathrm{~Hz}, \mathrm{H}-6), 3.44$ (dd, $\left.1 \mathrm{H}, J=2.6 \mathrm{~Hz}, \mathrm{H}-6^{\prime}\right) 3.36,3.28,3.12$ (3t, 3H, $J=9.2 \mathrm{~Hz}, \mathrm{H}-2 \mathrm{H}-3$ $\mathrm{H}-4), 2.52$ (ddd, $1 \hat{\mathrm{H}, \mathrm{H}-5)}{ }^{13} \mathrm{C}$ NMR $\left(90 \mathrm{MHz}, \mathrm{D}_{2} \mathrm{O}\right) \delta 148.3(\mathrm{C}=\mathrm{N})$, $139.7,132.8,132.7,132.6$ (s, 2C), 129.8 (s, 2C), 129.6, 119.6, 111.5, 83.3(C-1), 80.1, 77.2, 72.6,69.2 (4s, C-2 C-3 C-4C-5), 60.5 (C-6); Anal. Calcd for $\mathrm{C}_{17} \mathrm{H}_{19} \mathrm{NO}_{6} \mathrm{~S}$ (365.41): C, 55.88; $\mathrm{H}, 5.24 ; \mathrm{N}, 3.83 ; \mathrm{S}, 8.77$. Found: C, 55.74; H, 5.06; N, 3.52; $\widehat{\mathrm{S}}, 8.55$. 
4.3.6. 1-S-(Z)-Isonicotinoyl-hydroximoyl-1-thio- $\beta-\mathrm{D}-$ glucopyranose $2 \mathrm{i}$

A solution composed of $\mathbf{1 i}(90 \mathrm{mg}, 0.18 \mathrm{mmol})$ in a mixture of $\mathrm{MeOH}(2 \mathrm{~mL})$, water $(2 \mathrm{~mL})$ and $\mathrm{NEt}_{3}(0.4 \mathrm{~mL})$ was treated according to general procedure $\mathrm{B}$. The residue was purified (RP-18: water) to afford $2 \mathbf{i}$ ( $53 \mathrm{mg}, 0.16 \mathrm{mmol}, 90 \%$ yield) as a white solid.

$R_{\mathrm{f}}=0.48(\mathrm{EtOAc} / \mathrm{MeOH}, 4: 1) ;[\alpha]_{\mathrm{D}}^{20}-1.6\left(\right.$ c $\left.1, \mathrm{H}_{2} \mathrm{O}\right) ; \mathrm{mp}=140{ }^{\circ} \mathrm{C}$ darkening, $175^{\circ} \mathrm{C}$ decomposition; ${ }^{1} \mathrm{H}$ NMR $\left(300 \mathrm{MHz}, \mathrm{D}_{2} \mathrm{O}\right) \delta 8.62$ (dd, $2 \mathrm{H}, J=1.5$ and $5.5 \mathrm{~Hz}, \mathrm{H}-\mathrm{ar}$ ), 7.57 (dd, 2H, H-ar), 4.33 (d, $1 \mathrm{H}$, $J=9.8 \mathrm{~Hz}, \mathrm{H}-1$ ), 3.60 (dd, $1 \mathrm{H}, J=2.4$ and $12.7 \mathrm{~Hz}, \mathrm{H}-6$ ), 3.52 (dd, $\left.1 \mathrm{H}, J=4.9 \mathrm{~Hz}, \mathrm{H}-6^{\prime}\right), 3.40(\mathrm{t}, 2 \mathrm{H}, J=9.2 \mathrm{~Hz}, \mathrm{H}-2), 3.31(\mathrm{t}, 1 \mathrm{H}$, $J=9.0 \mathrm{~Hz}, \mathrm{H}-4), 3.25$ (t, 1H, J=8.7 Hz, H-3), 2.75 (ddd, 1H, H-5); ${ }^{13} \mathrm{C}$ NMR (75 MHz, D $\left.\mathrm{D}_{2} \mathrm{O}\right) \delta 152.7(\mathrm{C}=\mathrm{N}), 149.6$ (s, 2C, CH-ar), 141.6, 124.3 (s, 2C, CH-ar), 83.1 (C-1), 80.3 (C-5), 77.3 (C-3), 72.5, 69.2 (2s, C-2, C-4), 60.5 (C-6); Anal. Calcd for $\mathrm{C}_{12} \mathrm{H}_{16} \mathrm{~N}_{2} \mathrm{O}_{6} \mathrm{~S}, 0.5$ $\mathrm{H}_{2} \mathrm{O}$ : C, 44.30; $\mathrm{H}, 5.27 ; \mathrm{N}, 8.61 ; \mathrm{O} ; 31.97 ; \mathrm{S}, 9.86$. Found: $\mathrm{C}$, $44.34 ; \mathrm{H}, 5.26$; N, 8.62; O, 32.45 .

\subsection{Spiro-cyclization}

4.4.1. (1S)- and (1R)-2,3,4,6-Tetra- 0 -acetyl-1,5-anhydro-Dglucitol-spiro[1.5]-3-(phenyl)-1,4,2-oxathiazole (1S)-3a and (1R)-3a

When the spiro-cyclization was carried out with $\mathbf{1 a}(278 \mathrm{mg})$ in $\mathrm{CHCl}_{3}$ in the presence of NBS (2 equiv) and DBU (1 equiv) according to the general procedure $C$, TLC suggested a $\sim 1: 1$ ratio of the $(1 S) /$ $(1 R)$-epimers. Separation by repeated column chromatographies with a gradient of PE/EtOAc as the mobile phase afforded (1S)-3a (44 mg, 16\%) and (1R)-3a (51 mg, 18\%).

4.4.2. (1S)-2,3,4,6-Tetra-O-acetyl-1,5-anhydro-D-glucitolspiro[1.5]-3-(4-nitrophenyl)-1,4,2-oxathiazole (1S)-3b

A solution composed of $\mathbf{1 b}(827 \mathrm{mg}, 1.56 \mathrm{mmol})$ and NBS (557 mg, $3.13 \mathrm{mmol}$ ) was treated according to the general procedure $\mathrm{C}$ to afford a first crop of pure (1S)-3b (259 $\mathrm{mg})$ as a white foam and an additional crop (144 mg) of a mixture of (1S)-3b and ( $1 R)$-3b in a 7:93 ratio, respectively, ( ${ }^{1} \mathrm{H}$ NMR). The calculated yields were, respectively, $33 \%$ and $16 \%$ for the $(1 S)$ - and $(1 R)$-epimers.

$R_{\mathrm{f}}=0.62(\mathrm{PE} /$ EtOAc, $3: 2) ;[\alpha]_{\mathrm{D}}^{20}+33\left(\mathrm{c} 1, \mathrm{CH}_{2} \mathrm{Cl}_{2}\right) ;{ }^{1} \mathrm{H}$ NMR $\left(300 \mathrm{MHz}, \mathrm{CDCl}_{3}\right) \delta 8.31(\mathrm{~d}, 2 \mathrm{H}, J=9.0 \mathrm{~Hz}, \mathrm{H}-\mathrm{ar}), 7.87(\mathrm{~d}, 2 \mathrm{H}$, $J=9.0 \mathrm{~Hz}, \mathrm{H}-\mathrm{ar}), 5.64(\mathrm{~m}, 2 \mathrm{H}, \mathrm{H}-2 \mathrm{H}-3), 5.29$ (m, 1H, H-4), 4.44 (ddd, $1 \mathrm{H}, J=2.1,3.6$ and $10.3 \mathrm{~Hz}, \mathrm{H}-5$ ), 4.35 (dd, $1 \mathrm{H}, J=3.6$ and $12.7 \mathrm{~Hz}, \mathrm{H}-6^{\prime}$ ), 4.10 (dd, $1 \mathrm{H}, J=2.1$ and $12.7 \mathrm{~Hz}, \mathrm{H}-6$ ), 2.05, 2.07, 2.09, 2.10 (4s, $\left.12 \mathrm{H}, \mathrm{CH}_{3} \mathrm{CO}\right) ;{ }^{13} \mathrm{C} \mathrm{NMR}\left(75 \mathrm{MHz}, \mathrm{CDCl}_{3}\right) \delta 170.4$ 169.5, 169.3, 169.2, (4s, $\left.\mathrm{CH}_{3} \mathrm{CO}\right), 154.4(\mathrm{C}=\mathrm{N}), 149.3,132.8,128.8$ (s, 2C, CH-ar), 124.1 (s, 2C, CH-ar), 123.4 (C-1), 70.9 (C-2 or C-3), 70.8 (C-5), 67.8 (C-2 or C-3), 67.2 (C-4), 60.9 (C-6), 20.6 (s, $\mathrm{CH}_{3} \mathrm{CO}$ ), $20.4\left(\mathrm{~s}, 3 \mathrm{C}, \mathrm{CH}_{3} \mathrm{CO}\right)$; MS (ESI $\left.>0\right) \mathrm{m} / z=549.0[\mathrm{M}+\mathrm{Na}]^{+}, 1074.5$ $[2 \mathrm{M}+\mathrm{Na}]^{+}$; HRMS (ESI >0) $\mathrm{m} / z=\mathrm{C}_{21} \mathrm{H}_{22} \mathrm{~N}_{2} \mathrm{NaO}_{12} \hat{\mathrm{S}}[\mathrm{M}+\mathrm{Na}]^{+}$calcd 549.0ิ791, found 549.0ิ92.

4.4.3. (1S)-2,3,4,6-Tetra-O-acetyl-1,5-anhydro-D-glucitolspiro[1.5]-3-(4-cyanophenyl)-1,4,2-oxathiazole (1S)-3c

A solution composed of 1 c $(680 \mathrm{mg}, 1.34 \mathrm{mmol})$ and NBS (476 mg, $2.67 \mathrm{mmol}$ ) was treated according to the general procedure $C$ to afford a first crop of pure (1S)-3c (103 mg, 15\%) as a white foam and an additional crop (48 $\mathrm{mg}$ ) of a mixture of (1S)-3c and $(1 R)-3 \mathrm{c}$ in a 5:95 ratio, respectively, $\left({ }^{1} \mathrm{H} \mathrm{NMR}\right)$. The calculated yields were, respectively, $15 \%$ and $7 \%$ for the $(1 S)$ - and $(1 R)$ epimers.

$R_{\mathrm{f}}=0.49(\mathrm{PE} / \mathrm{EtOAc}, 3: 2) ;[\alpha]_{\mathrm{D}}^{20}+35\left(\mathrm{c} 1, \mathrm{CH}_{2} \mathrm{Cl}_{2}\right) ;{ }^{1} \mathrm{H}$ NMR $\left(300 \mathrm{MHz}, \mathrm{CDCl}_{3}\right) \delta 7.79(\mathrm{~d}, 2 \mathrm{H}, J=8.7 \mathrm{~Hz}, \mathrm{CH}-\mathrm{ar}), 7.74(\mathrm{~d}, 2 \mathrm{H}$, $J=8.7 \mathrm{~Hz}, \mathrm{CH}-\mathrm{ar}), 5.28$ (m, 1H, H-4), 5.64 (m, 2H, H-2 H-3), 4.44 (ddd, $1 \mathrm{H}, J=2.1,3.6$ and $10.3 \mathrm{~Hz}, \mathrm{H}-5$ ), 4.33 (dd, $1 \mathrm{H}, J=3.6$ and $\left.12.7 \mathrm{~Hz}, \mathrm{H}-6^{\prime}\right), 4.10(\mathrm{dd}, 1 \mathrm{H}, J=2.1$ and $12.7 \mathrm{~Hz}, \mathrm{H}-6), 2.09(\mathrm{~s}, 6 \mathrm{H}$, $\left.\mathrm{CH}_{3} \mathrm{CO}\right), \hat{2} .04,2.06\left(2 \mathrm{~s}, 6 \mathrm{H}, \mathrm{CH}_{3} \mathrm{CO}\right) ;{ }^{13} \mathrm{C} \mathrm{NMR}\left(75 \mathrm{MHz}, \mathrm{CDCl}_{3}\right) \delta$
170.4, 169.6, 169.29, 169.26, (4s, $\left.\mathrm{CH}_{3} \mathrm{CO}\right), 154.5(\mathrm{C}=\mathrm{N}), 132.6(\mathrm{~s}$, 2C, CH-ar), 131.2, 128.4 (s, 2C, CH-ar), 123.3 (C-1), 117.7, 115.1 $(\mathrm{C} \equiv \mathrm{N}), 70.84$ (C-2 or C-3), $70.80(\mathrm{C}-5), 67.9$ (C-2 or C-3), 67.3 (C4), 60.9 (C-6), $20.6\left(\mathrm{CH}_{3} \mathrm{CO}\right), 20.4$ (s, 3C, $\left.\mathrm{CH}_{3} \mathrm{CO}\right)$; MS (ESI $\left.>0\right) \mathrm{m} /$ $z=529.0 \quad[\mathrm{M}+\mathrm{Na}]^{+}, \quad 1034.5 \quad[2 \mathrm{M}+\mathrm{Na}]^{+} ;$HRMS (ESI $\left.>0\right) \quad \mathrm{m} /$ $z=\mathrm{C}_{22} \mathrm{H}_{22} \mathrm{~N}_{2} \mathrm{NaO}_{10} \mathrm{~S}$ [N $\left.\mathrm{M}+\mathrm{Na}\right]^{+}$calcd 529.0893, found 529.0898.

4.4.4. (1S)-2,3,4,6-Tetra-O-acetyl-1,5-anhydro-D-glucitolspiro[1.5]-3-(4-methoxyphenyl)-1,4,2-oxathiazole (1S)-3e

A solution composed of 1d (690 mg, $1.34 \mathrm{mmol})$ and NBS (478 mg, $2.68 \mathrm{mmol}$ ) was treated according to the general procedure $\mathrm{C}$ to afford a first crop of pure (1S)-3e (372 $\mathrm{mg})$ as a white foam and an additional crop (218 $\mathrm{mg}$ ) of a mixture of (1S)-3e and (1R)-3e in a 45:55 ratio, respectively, $\left({ }^{1} \mathrm{H}\right.$ NMR). The calculated yields were, respectively, $69 \%$ and $17 \%$ for the $(1 S)$ - and $(1 R)$-epimers.

$R_{\mathrm{f}}=0.43\left(\mathrm{PE} /\right.$ EtOAc, 3:2); $[\alpha]_{\mathrm{D}}^{20}+52\left(\mathrm{c} 1, \mathrm{CH}_{2} \mathrm{Cl}_{2}\right) ;{ }^{1} \mathrm{H} \mathrm{NMR}$ $\left(300 \mathrm{MHz}, \mathrm{CDCl}_{3}\right) \delta 7.61(\mathrm{~d}, 2 \mathrm{H}, J=8.9 \mathrm{~Hz}, \mathrm{CH}-\mathrm{ar}), 6.94(\mathrm{~d}, 2 \mathrm{H}$, $J=8.9 \mathrm{~Hz}, \mathrm{CH}-\mathrm{ar}), 5.62(\mathrm{~m}, 2 \mathrm{H}, \mathrm{H}-2 \mathrm{H}-3), 5.26(\mathrm{~m}, 1 \mathrm{H}, \mathrm{H}-4), 4.41$ (ddd, $1 \mathrm{H}, J=2.0,3.6$ and $10.3 \mathrm{~Hz}, \mathrm{H}-5$ ), 4.34 (dd, $1 \mathrm{H}, J=3.6$ and $\left.12.6 \mathrm{~Hz}, \mathrm{H}-6^{\prime}\right), 4.07$ (dd, $1 \mathrm{H}, J=2.0$ and $\left.12.6 \mathrm{~Hz}, \mathrm{H}-6\right), 3.85(\mathrm{~s}, 3 \mathrm{H}$, OMe), 2.09, 2.08, 2.05, 2.03, (4s, $\left.12 \mathrm{H}, \mathrm{CH}_{3} \mathrm{CO}\right) ;{ }^{13} \mathrm{C}$ NMR $(75 \mathrm{MHz}$, $\left.\mathrm{CDCl}_{3}\right) \delta 170.6,169.6,169.5,169.4\left(4 \mathrm{~s}, \mathrm{CH}_{3} \mathrm{CO}\right), 162.2,155.9$ $(\mathrm{C}=\mathrm{N}), 129.6$ (s, 2C, CH-ar), $122.1(\mathrm{C}-1), 119.3,114.3(\mathrm{~s}, 2 \mathrm{C}, \mathrm{CH}-$ ar), 71.1 (C-2 or C-3), 70.5 (C-5), 67.9 (C-2 or C-3), 67.5 (C-4), 61.1 (C-6), 55.4 (OMe), $20.7\left(\mathrm{CH}_{3} \mathrm{CO}\right), 20.5$ (s, 3C, $\left.\mathrm{CH}_{3} \mathrm{CO}\right)$; MS $(\mathrm{ESI}>0) \quad m / z=511.8[\mathrm{M}+\mathrm{H}]^{+}, 534.0[\mathrm{M}+\mathrm{Na}]^{+}, 1022.4[2 \mathrm{M}+\mathrm{H}]^{+}$ $1044.6[2 \mathrm{M}+\mathrm{Na}]^{+}$; HRMS (ESI $\left.>0\right) \quad m / z=\mathrm{C}_{22} \mathrm{H}_{25} \mathrm{NNaO}_{11} \mathrm{~S}[\mathrm{M}+\mathrm{Na}]^{+}$ calcd 534.1046, found 534.1049.

4.4.5. (1R)-2,3,4,6-Tetra-O-acetyl-1,5-anhydro-D-glucitolspiro[1.5]-3-(3,4,5-trimethoxyphenyl)-1,4,2-oxathiazole (1R)-3f

Spiro-cyclization of $\mathbf{1 f}(580 \mathrm{mg})$ was attempted as before (NBS: $360 \mathrm{mg}$, 2 equiv; $\mathrm{CHCl}_{3}, 25 \mathrm{~mL}$ ) upon heating with a $60 \mathrm{~W}$ tungsten lamp for $1 \mathrm{~h}$. TLC showed that the transformation was not selective, yielding a multicomponent mixture. Workup and flash column chromatography (PE then PE/EtOAc 1:1) of the crude product $(827 \mathrm{mg}$ ) afforded four fractions (90, 33, 21, $41 \mathrm{mg})$. Only the third one was pure enough and led to exploitable NMR spectra (COSY, HSQC), concluding to the presence of (1R)-3f.

${ }^{1} \mathrm{H}$ NMR (300 MHz, $\left.\mathrm{CDCl}_{3}\right) \delta 6.93(\mathrm{~s}, 2 \mathrm{H}, \mathrm{CH}-\mathrm{ar}), 5.59(\mathrm{~d}, 1 \mathrm{H}$, $J=10.2 \mathrm{~Hz}, \mathrm{H}-2), 5.27(\mathrm{t}, 1 \mathrm{H}, \mathrm{H}-4), 5.10(\mathrm{dd}, 1 \mathrm{H}, J=9.5$ and $10.2 \mathrm{~Hz}, \mathrm{H}-3$ ), 4.35 (dd, $1 \mathrm{H}, J=3.3$ and $12.3 \mathrm{~Hz}, \mathrm{H}-6), 4.10(\mathrm{~m}, 2 \mathrm{H}$, $\left.\mathrm{H}-5 \mathrm{H}^{\prime} 6^{\prime}\right), 3.90$ (s, 9H, OMe), 2.08, 2.07 (2s, 6H, $\left.\mathrm{CH}_{3} \mathrm{CO}\right), 2.05$ (s, $\left.6 \mathrm{H}, \widehat{\mathrm{CH}}_{3} \mathrm{CO}\right) ;{ }^{13} \mathrm{C}$ NMR $\left(75 \mathrm{MHz}, \mathrm{CDCl}_{3}\right) \delta 171.0,170.3,169.6$, 168.9, (4s, $\left.\mathrm{CH}_{3} \mathrm{CO}\right), 155.6(\mathrm{C}=\mathrm{N}), 153.8(\mathrm{~s}, 2 \mathrm{C}), 141.4,127.4(\mathrm{C}-1)$, 122.8, 105.7 (s, 2C, CH-ar), 73.7 (C-3), 71.0 (C-5), 68.7 (C-2), 67.5 (C-4), 61.42 (C-6), 61.40 (OMe), 56.7 (s, 2C, OMe), $20.7\left(\mathrm{CH}_{3} \mathrm{CO}\right)$, 20.6 (s, 2C, $\left.\mathrm{CH}_{3} \mathrm{CO}\right), 20.5\left(\mathrm{CH}_{3} \mathrm{CO}\right)$.

4.4.6. (1S)-2,3,4,6-Tetra-O-acetyl-1,5-anhydro-D-glucitolspiro[1.5]-3-[(4-phenyl)-phenyl]-1,4,2-oxathiazole (1S)-3g

A solution composed of $\mathbf{1 g}(200 \mathrm{mg}, 357 \mu \mathrm{mol})$ and NBS (127 mg, $715 \mu \mathrm{mol}$ ) was treated according to the general procedure $C$ to afford a first crop of pure (1S)-3g (65 mg) as a white foam and an additional crop (90 $\mathrm{mg}$ ) of a mixture of (1S)-3g and (1R)-3g in a 3:2 ratio, respectively. The calculated yields were, respectively, $61 \%$ and $18 \%$ for the $(1 S)$ - and $(1 R)$-epimers.

$R_{\mathrm{f}}=0.52\left(\mathrm{PE} /\right.$ EtOAc, 3:2); $[\alpha]_{\mathrm{D}}^{20}+45\left(\mathrm{c} 1, \mathrm{CH}_{2} \mathrm{Cl}_{2}\right) ;{ }^{1} \mathrm{H} \mathrm{NMR}$ $\left(300 \mathrm{MHz}, \mathrm{CDCl}_{3}\right) \delta 7.74(\mathrm{~d}, 2 \mathrm{H}, J=8.5 \mathrm{~Hz}, \mathrm{CH}-\mathrm{ar}), 7.65(\mathrm{~d}, 2 \mathrm{H}$, $J=8.5 \mathrm{~Hz}, \mathrm{CH}-\mathrm{ar}), 7.57-7.63(\mathrm{~m}, 2 \mathrm{H}, \mathrm{CH}-\mathrm{ar}), 7.37-7.50(\mathrm{~m}, 3 \mathrm{H}$, CH-ar), 5.64 (m, 2̂े, H-2 H-3), 5.28 (m, 1H, H-4), 4.44 (ddd, $1 \mathrm{H}$, $J=2.0,3.7$ and $10.3 \mathrm{~Hz}, \mathrm{H}-5), 4.36(\mathrm{dd}, 1 \mathrm{H}, J=3.7$ and $12.6 \mathrm{~Hz}, \mathrm{H}-$ $\left.6^{\prime}\right), 4.09(\mathrm{dd}, 1 \mathrm{H}, J=2.0$ and $12.6 \mathrm{~Hz}, \mathrm{H}-6), 2.09,2.06(2 \mathrm{~s}, 6 \mathrm{H}$, $\mathrm{CH}_{3} \mathrm{CO}$ ), 2.04 (2s, $\left.6 \mathrm{H}, \mathrm{CH}_{3} \mathrm{CO}\right) ;{ }^{13} \mathrm{C} \mathrm{NMR}\left(75 \mathrm{MHz}, \mathrm{CDCl}_{3}\right) \delta 170.5$, 169.6, 169.5, $169.4\left(4 \mathrm{~s}, \mathrm{CH}_{3} \mathrm{CO}\right), 156.1(\mathrm{C}=\mathrm{N}), 144.5,139.6,128.9$ (s, 2C, CH-ar), 128.4 (s, 2C, CH-ar), 128.1 (CH-ar), 127.5 (s, 2C, 
CH-ar), 127.1 (s, 2C, CH-ar), 125.8, 122.4 (C-1), 71.0 (C-2 or C-3), 70.6 (C-5), 68.0 (C-2 or C-3), 67.5 (C-4), 61.1 (C-6), $20.7\left(\mathrm{CH}_{3} \mathrm{CO}\right)$, 20.5 (s, 3C, $\left.\mathrm{CH}_{3} \mathrm{CO}\right)$; $\mathrm{MS}(\mathrm{ESI}>0) \quad \mathrm{m} / z=557.8[\mathrm{M}+\mathrm{H}]^{+}, \quad 580.0$ $[\mathrm{M}+\mathrm{Na}]^{+}, 1114.4[2 \mathrm{M}+\mathrm{H}]^{+}, 1136.7[2 \mathrm{M}+\mathrm{Na}]^{+}$; HRMS $(\mathrm{ESI}>0) \mathrm{m} /$ $z=\mathrm{C}_{27} \mathrm{H}_{27} \mathrm{NNaO}_{10} \mathrm{~S}[\mathrm{M}+\mathrm{Na}]^{+}$calcd 580.1253, found 580.1252.

4.4.7. (1S)-2,3,4,6-Tetra-O-acetyl-1,5-anhydro-D-glucitolspiro[1.5]-3-(2-naphthyl)-1,4,2-oxathiazole (1S)-3h ${ }^{23}$

A solution composed of $\mathbf{1 h}(655 \mathrm{mg}, 1.23 \mathrm{mmol})$ and NBS (437 mg, $2.46 \mathrm{mmol}$ ) was treated according to the general procedure $C$ to afford a first crop of pure (1S)-3h (181 mg) as a white foam and an additional crop (160 mg) of a mixture of (1S)-3h and $(1 R)-3 \mathbf{h}$ in a 1:2 xatio, respectively. The calculated yields xere, respectively, $36 \%$ and $16 \%$ for the $(1 S)$ - and $(1 R)$-epimers.

4.4.8. (1R)-2,3,4,6-Tetra- 0 -acetyl-1,5-anhydro-D-glucitolspiro[1.5]-3-(4-pyridyl)-1,4,2-oxathiazole (1R)-3i

A solution composed of $\mathbf{1 i}(636 \mathrm{mg}, 1.31 \mathrm{mmol})$ and NBS (467 mg, $2.62 \mathrm{mmol}$ ) in anhydrous chloroform $(30 \mathrm{~mL}$ ) was treated with for $30 \mathrm{~min}$ according to the general procedure C. TLC monitoring showed the appearance of two main products (more mobile than 1i) and two minor products. Workup and flash chromatography with petroleum ether EtOAc (containing 0.1\% $\mathrm{Et}_{3} \mathrm{~N}$ ) 7:3 then $2: 3$ afforded a mixture $(107 \mathrm{mg}$ ) with 3 components (NMR) some being brominated (as shown by TLC using a specific staining reagent with fluorescein), and (1R)-3i $(190 \mathrm{mg}, 0.39 \mathrm{mmol}, 30 \%$ yield) as a transparent solid which appeared labile at room temperature and was kept at $0{ }^{\circ} \mathrm{C}$.

$R_{\mathrm{f}}=0.63$ (EtOAc); $\mathrm{mp}=60-61^{\circ} \mathrm{C} ;[\alpha]_{\mathrm{D}}^{20}+181.7\left(\mathrm{c} \mathrm{0.9}, \mathrm{CH}_{2} \mathrm{Cl}_{2}\right) ;{ }^{1} \mathrm{H}$ $\operatorname{NMR}\left(300 \mathrm{MHz}, \mathrm{CDCl}_{3}\right) \delta 8.75$ (dd, $2 \mathrm{H}, J=1.6$ and $\left.4.4 \mathrm{~Hz}, \mathrm{H}-\mathrm{ar}\right), 7.57$ (dd, 2H, H-ar), 5.63 (d, $1 \mathrm{H}, J=10.2 \mathrm{~Hz}, \mathrm{H}-2), 5.28$ (t, 1H, $J=9.7 \mathrm{~Hz}$, $\mathrm{H}-4), 5.11(\mathrm{dd}, 1 \mathrm{H}, J=9.5 \mathrm{~Hz}, \mathrm{H}-3), 4.34(\mathrm{dd}, 1 \mathrm{H}, J=4.2$ and $12.9 \mathrm{~Hz}$, $\mathrm{H}-6$ ), 4.12 (m, 2H, H-5 H-6'), 2.08, 2.07, 2.06, 2.05 (4s, 12 $\mathrm{H}^{\mathrm{C}} \mathrm{CH}_{3} \mathrm{CO}$ ); ${ }^{13} \mathrm{C}$ NMR $\left(75 \mathrm{MHz}, \mathrm{CDCl}_{3}\right) \delta 170.9,170.3,169.5,168.9\left(4 \mathrm{~s}, \mathrm{CH}_{3} \mathrm{CO}\right)$, $154.2(\mathrm{C}=\mathrm{N}), 151.0$ (s, 2C, CH-ar), 135.2, 128.3 (C-1), 122.0 (s, 2C, CH-ar), 73.5 (C-3), 71.1 (C-5), 68.6 (C-2), 67.3 (C-4), 61.3 (C-6), 21.1, 21.0, 20.90, 20.92 (4s, $\left.\mathrm{CH}_{3} \mathrm{CO}\right)$; Anal. Calcd for $\mathrm{C}_{20} \mathrm{H}_{22} \mathrm{~N}_{2} \mathrm{O}_{10} \mathrm{~S}$ (482.46): C, 49.79; H, 4.60; N, 5.81; O, 33.16. Found: C, 50.07; H, 4.68 ; N, 5.55; 0, 33.41 .

\subsection{Deacetylation of spiro-oxathiazoles}

4.5.1. (1S)-1,5-Anhydro-D-glucitol-spiro[1.5]-3-(4-nitrophenyl)1,4,2-oxathiazole (1S)-4b

A solution composed of (1S)-3b (255 mg) was treated according to general procedure D to afford (1S)-4b $(124 \mathrm{mg}, 71 \%)$ as a pale yellow solid.

$R_{\mathrm{f}}=0.43 \quad\left(\right.$ EtOAc/MeOH, 85:15); $[\alpha]_{\mathrm{D}}^{20}+58.8$ (c 1, DMSO); $\mathrm{mp}=182-183^{\circ} \mathrm{C} ;{ }^{1} \mathrm{H}$ NMR $\left(300 \mathrm{MHz}, \mathrm{CD}_{3} \mathrm{OD}, 50{ }^{\circ} \mathrm{C}\right) \delta 8.30(\mathrm{~d}$, $2 \mathrm{H}, \hat{J}=8.9 \mathrm{~Hz}, \mathrm{CH}-\mathrm{ar}$ ), 7.90 (d, $2 \mathrm{H}, J=8.9 \mathrm{~Hz}, \mathrm{CH}-\mathrm{ar}$ ), 3.92 (ddd, $1 \mathrm{H}, J=2.3,4.7$ and $9.5 \mathrm{~Hz}, \mathrm{H}-5), 3.90(\mathrm{~d}, 1 \mathrm{H}, J=9.5 \mathrm{~Hz}, \mathrm{H}-2), 3.82$ (dd, $1 \mathrm{H}, J=2.3$ and $\left.12.2 \mathrm{~Hz}, \mathrm{H}-6^{\prime}\right), 3.78(\mathrm{t}, 1 \mathrm{H}, J=9.5 \mathrm{~Hz}, \mathrm{H}-3$ ), $3.76(\mathrm{dd}, 1 \mathrm{H}, J=4.7$ and $12.2 \mathrm{~Hz}, \mathrm{H}-6), 3.53(\mathrm{t}, 1 \mathrm{H}, J=9.5 \mathrm{~Hz}, \mathrm{H}-$ 4); ${ }^{13} \mathrm{C}$ NMR ( $\left.75 \mathrm{MHz}, \mathrm{CD}_{3} \mathrm{OD}, 50{ }^{\circ} \mathrm{C}\right) \delta 154.5(\mathrm{C}=\mathrm{N}), 150.7,135.3$, 129.8 (s, 2C, CH-ar), 129.0, 125.2 (s, 2C, CH-ar), 77.7 (C-5), 76.2 (C-3), 73.0 (C-2), 70.8 (C-4), 62.2 (C-6); MS (ESI <0) $\mathrm{m} / z=392.9$ $[\mathrm{M}+\mathrm{Cl}]^{-}, 750.5[2 \mathrm{M}+\mathrm{Cl}]^{-} ;$HRMS $(\mathrm{ESI}<0) \quad \mathrm{m} / z=\mathrm{C}_{13} \mathrm{H}_{14} \mathrm{ClN}_{2} \mathrm{O}_{8} \mathrm{~S}$ $[\mathrm{M}+\mathrm{Cl}]$ - calcd 393.0159, found 393.0158.

\subsection{2. (1S)-1,5-Anhydro-D-glucitol-spiro[1.5]-3-(4-}

cyanophenyl)-1,4,2-oxathiazole (1S)-4c

A solution composed of (1S)-3c (100 mg) was treated according to general procedure D to afford (1S)-4c (56 mg, $84 \%$ ) as a pale yellow foam.

$R_{\mathrm{f}}=0.35(\mathrm{EtOAc} / \mathrm{MeOH}, 85: 15) ;[\alpha]_{\mathrm{D}}^{20}+36.3$ (c 1, DMSO); ${ }^{1} \mathrm{H}$ NMR (300 MHz, CD $\left.\mathrm{CD}_{3} \mathrm{OD}\right) \delta 7.85$ (m, 4H, CH-ar), 3.86-3.93 (m, $\left.1 \mathrm{H}, \mathrm{H}-5\right)$, $3.89(\mathrm{~d}, 1 \mathrm{H}, J=9.6 \mathrm{~Hz}, \mathrm{H}-2), 3.81$ (dd, $1 \mathrm{H}, J=2.3$ and $12.3 \mathrm{~Hz}$, H$\left.6^{\prime}\right)$, 3.70-3.78 (m, 2H, H-3 H-6), 3.51 (t, $\left.1 \mathrm{H}, J=9.0 \mathrm{~Hz}, \mathrm{H}-4\right) ;{ }^{13} \mathrm{C}$ $\operatorname{NMR}\left(75 \mathrm{MHz}, \mathrm{CD}_{3} \mathrm{OD}\right) \delta 155.8(\mathrm{C}=\mathrm{N}), 134.0$ (s, 2C, CH-ar), 133.7, 129.4 (s, 2C, CH-ar), 128.8 (C-1), 119.0, 115.6 (CN), 77.6 (C-5), 76.1 (C-3), 72.7 (C-2), $70.6(\mathrm{C}-4), 61.9(\mathrm{C}-6)$; MS (ESI <0) m/ $z=372.9[\mathrm{M}+\mathrm{Cl}]_{\wedge}^{-} ;$HRMS $(\mathrm{ESI}<0) \quad m / z=\mathrm{C}_{14} \mathrm{H}_{14} \mathrm{ClN}_{2} \mathrm{O}_{6} \mathrm{~S} \quad[\mathrm{M}+\mathrm{Cl}]_{\wedge}^{-}$ calcd 373.0261, found 373.0264.

4.5.3. (1S)-1,5-Anhydro-D-glucitol-spiro[1.5]-3-(4-fluorophenyl)-1,4,2-oxathiazole (1S)-4d

Prepared by general procedure D from (1S)-3d (500 mg) to afford (1S)-4d (392 $\mathrm{mg}, 79 \%$ ) as a pale yellow solid.

$R_{\mathrm{f}}=0.69\left(\mathrm{CHCl}_{3} / \mathrm{MeOH}, 7: 3\right) ;[\alpha]_{\mathrm{D}}^{20}+51$ (c 1.1, DMSO); $\mathrm{np}=192-$ $193{ }^{\circ} \mathrm{C}$; ${ }^{1} \mathrm{H}$ NMR (360 MHz, DMSO-d $\left.{ }_{6}\right) \delta 7.73-7.69$ (m, 2H, H-ar), 7.38-7.33 (m, 2H, H-ar), 3.72-3.62 (m, 3H, H-6 H-6' H-1), 3.533.23 (m, 3H, H-2 H-3 H-5); ${ }^{13} \mathrm{C} \mathrm{NMR}\left(90 \mathrm{MHz}\right.$, DMSO- $\left.\mathrm{d}_{6}\right) \delta 163.2$, $154.7(\mathrm{C}=\mathrm{N}), 130.8,130.7,128.3,117.5,117.2,108.8(\mathrm{C}-1), 77.7$, 75.3, 72.1, 70.1 (C-2 C-3 C-4 C-5), 61.2 (C-6); Anal. Calcd for $\mathrm{C}_{13} \mathrm{H}_{14} \mathrm{NO}_{6} \mathrm{~S}$ (331.32): C.47.13; H, 4.26; N, 4.23; S, 9.68. Found: C, 47.33; H, 4.09; N, 4.52; S, 9.45 .

4.5.4. (1S)-1,5-Anhydro-D-glucitol-spiro[1.5]-3-(4-methoxyphenyl)-1,4,2-oxathiazole (1S)-4e

A solution composed of (1S)-3e (370 mg) was treated according to general procedure D to afford (1S)-4e (196 $\mathrm{mg}, 79 \%)$ as a pale yellow solid.

$R_{\mathrm{f}}=0.33(\mathrm{EtOAc} / \mathrm{MeOH}, 85: 15) ;[\alpha]_{\mathrm{D}}^{20}+43.4$ (c 1, DMSO); $\mathrm{mp}=192-194{ }^{\circ} \mathrm{C} ;{ }^{1} \mathrm{H}$ NMR $\left(300 \mathrm{MHz}\right.$, DMSO $\left.-d_{6}+\varepsilon \mathrm{D}_{2} \mathrm{O}\right) \delta 7.57(\mathrm{~d}$, $2 \mathrm{H}, \hat{J}=8.7 \mathrm{~Hz}, \mathrm{H}-\mathrm{ar}$ ), 7.04 (d, $2 \mathrm{H}, J=8.7 \mathrm{~Hz}, \mathrm{H}-\mathrm{ar}), 3.80$ (s, 3H, OMe $), 3.43-3.71(\mathrm{~m}, 5 \mathrm{H}), 3.24(\mathrm{t}, 1 \mathrm{H}, J=9.4 \mathrm{~Hz}) ;{ }^{13} \mathrm{C}$ NMR (75 MHz, DMSO- $\left.d_{6}+\varepsilon \mathrm{D}_{2} \mathrm{O}\right) \delta 161.6,154.6,129.2$ (s, 2C, CH-ar), 126.8, 119.8, 114.8 (s, 2C, CH-ar), 76.6, 74.3, 71.0, 69.1, 60.3 (C6), $55.6(\mathrm{OMe})$; MS $(\mathrm{ESI}<0) \mathrm{m} / z=377.9[\mathrm{M}+\mathrm{Cl}]^{-}$; $\mathrm{MS}(\mathrm{ESI}>0) \mathrm{m} /$ $z=365.9 \quad[\mathrm{M}+\mathrm{Na}]^{+},{ }^{\lambda} \quad 708.8 \quad[2 \mathrm{M}+\mathrm{Na}]^{+} ; \stackrel{\text { HRMS }}{ } \quad($ ESI $>0) \quad \mathrm{m} /$ $z=\mathrm{C}_{14} \mathrm{H}_{17} \mathrm{NNaO}_{7} \mathrm{~S}[\mathrm{M}+\mathrm{Na}]^{+}$calcd 366.0623 , found 366.0621.

\subsection{5. (1S)-1,5-Anhydro-D-glucitol-spiro[1.5]-3-[(4-phenyl)-} phenyl]-1,4,2-oxathiazole (1S)-4g

A solution composed of (1S)-3g (65 mg) was treated according to general procedure D to afford ( $1 S)-\mathbf{4 g}$ (44 $\mathrm{mg}, 97 \%$ ) as a pale yellow foam.

$R_{\mathrm{f}}=0.32\left(\right.$ EtOAc/MeOH, 85:15); $[\alpha]_{\mathrm{D}}^{20}+39.4(c 0.6, \mathrm{DMSO}) ;{ }^{1} \mathrm{H}$ NMR $\left(500 \mathrm{MHz}, \mathrm{CD}_{3} \mathrm{OD}\right) \delta 7.79(\mathrm{~d}, 2 \mathrm{H}, J=8.3 \mathrm{~Hz}, \mathrm{CH}-\mathrm{ar}), 7.73(\mathrm{~d}, 2 \mathrm{H}$, $J=8.3 \mathrm{~Hz}, \mathrm{H}$-ar), $7.65-7.69$ ( $\mathrm{m}, 2 \mathrm{H}, \mathrm{H}-\mathrm{ar}$ ), 7.36-7.52 (m, 3H, CH-ar), 3.92 (ddd, $1 \mathrm{H}, J=2.3,4.6$ and $9.9 \mathrm{~Hz}, \mathrm{H}-5), 3.88(\mathrm{~d}, 1 \mathrm{H}, J=9.9 \mathrm{~Hz}$, $\mathrm{H}-2$ ), $3.82\left(\mathrm{dd}, 1 \mathrm{H}, J=2.3\right.$ and $\left.12.3 \mathrm{~Hz}, \mathrm{H}-6^{\prime}\right), 3.73-3.79(\mathrm{~m}, 2 \mathrm{H}, \mathrm{H}-3$ $\mathrm{H}-6), 3.52(\mathrm{t}, 1 \mathrm{H}, J=9.9 \mathrm{~Hz}, \mathrm{H}-4),{ }^{13} \mathrm{C} \mathrm{NMR}\left(125 \mathrm{MHz}, \mathrm{CD}_{3} \mathrm{OD}\right) \delta$ 62.0 (C-6), 70.8 (C-4), 72.8 (C-2), 76.2 (C-3), 77.5 (C-5), 127.8 (C-1), 128.1 (s, 2C, CH-ar), 128.5 (s, 2C, CH-ar), 129.2 (s, 2C, CH-ar), 129.3 (s, 2C, CH-ar), 130.1 (s, 2C, CH-ar), 133.8, 141.1, 145.4, 157.2 $(\mathrm{C}=\mathrm{N})$; $\mathrm{MS}(\mathrm{ESI}<0) \quad \mathrm{m} / \mathrm{z}=423.9 \quad[\mathrm{M}+\mathrm{Cl}]^{-}$; HRMS $(\mathrm{ESI}<0) \mathrm{m} /$

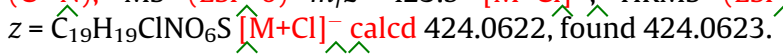

4.5.6. (1S)-1,5-Anhydro-D-glucitol-spiro[1.5]-3-(2-naphthyl)1,4,2-oxathiazole (1S)- $4 \mathrm{~h}^{23}$

A solution composed of (1S)-3h (180 mg) was treated according to general procedure D to afford (1S)-4h (116 mg, 94\%) as a pale yellow solid.

4.5.7. (1R)-1,5-Anhydro-D-glucitol-spiro[1.5]-3-(4-pyridyl)1,4,2-oxathiazole $(1 R)-4 i$

$(1 R)-3 \mathbf{i}(115 \mathrm{mg}, 0.24 \mathrm{mmol})$ was treated according to general procedure $\mathrm{B}\left(\mathrm{NEt}_{3}, \mathrm{MeOH}, \mathrm{H}_{2} \mathrm{O}\right)$ to afford $(1 R)-\mathbf{4 i}(71 \mathrm{mg}$, $0.22 \mathrm{mmol}, 95 \%$ yield) as a white solid.

$R_{\mathrm{f}}=0.40(\mathrm{EtOAc} / \mathrm{MeOH}, 4: 1) ;[\alpha]_{\mathrm{D}}^{20}-4$ (c 0.3, DMF); $\mathrm{mp}=174-$ $175^{\circ} \mathrm{C} ;{ }^{1} \mathrm{H}$ NMR $\left(300 \mathrm{MHz}, \mathrm{D}_{2} \mathrm{O}\right) \delta 8.63(\mathrm{dd}, 2 \mathrm{H}, \hat{\jmath=1.8} \widehat{\text { and }}$ 
$4.5 \mathrm{~Hz}, \mathrm{H}-\mathrm{ar}$ ), 7.70 (dd, 2H, H-ar), 3.94 (d, 1H,J = $10.2 \mathrm{~Hz}, \mathrm{H}-2$ ), 3.81 $\left(\mathrm{m}, 3 \mathrm{H}, \mathrm{H}-5 \mathrm{H}-6 \mathrm{H}-6^{\prime}\right), 3.60(\mathrm{t}, 1 \mathrm{H}, J=9.1 \mathrm{~Hz}, \mathrm{H}-4), 3.40(\mathrm{dd}, 2 \mathrm{H}$, $J=9.0 \mathrm{~Hz}, \mathrm{H}-3) ;{ }^{13} \mathrm{C}$ NMR $\left(75 \mathrm{MHz}, \mathrm{D}_{2} \mathrm{O}\right) \delta 156.2(\mathrm{C}=\mathrm{N}), 150.0(\mathrm{~s}$, 2C, CH-ar), 135.9, 130.8 (C-1), 122.6 (s, 2C, CH-ar), 76.7 (C-3), 75.5 (C-5), 71.6 (C-2), $68.5(\mathrm{C}-4), 60.2(\mathrm{C}-6)$; HRMS (FAB > 0) m/ $z=\mathrm{C}_{12} \mathrm{H}_{15} \mathrm{~N}_{2} \mathrm{O}_{6} \mathrm{~S}[\mathrm{M}+\mathrm{H}]^{+}$calcd 315.0651, found 315.0655.

\subsection{Synthesis of $\boldsymbol{N}$-glycosyl-amidoximes}

\subsection{1. $N$-(2,3,4,6-Tetra- $O$-acetyl- $\beta$-D-glucopyranosyl)-} benzamidoxime $8 \mathbf{a}$

Prepared according to general procedure E to afford 8a in $57 \%$ yield as a syrup. The product decomposed rapidly during the NMR spectroscopic measurement.

\subsection{2. $N$-(2,3,4,6-Tetra- 0 -acetyl- $\beta$-D-glucopyranosyl)-4- nitrobenzamidoxime $\mathbf{8 b}$}

Prepared according to general procedure $\mathrm{E}$ to afford $\mathbf{8 b}$ $(880 \mathrm{mg})$ in $64 \%$ yield.

$[\alpha]_{\mathrm{D}}+20\left(\right.$ c 1.2, $\left.\mathrm{CHCl}_{3}\right)$; mp $=183-185^{\circ} \mathrm{C} ;{ }^{1} \mathrm{H}$ NMR $(360 \mathrm{MHz}$, $\left.\mathrm{CDCl}_{3}\right) \hat{\delta} 9.36(\mathrm{~s}, 1 \mathrm{H}, \mathrm{OH}), 8.25(\mathrm{~d}, 2 \mathrm{H}, J=9.1 \mathrm{~Hz}, \mathrm{H}-\mathrm{ar}), 7.72(\mathrm{~d}$, $2 \mathrm{H}, J=8.4 \mathrm{~Hz}, \mathrm{H}-\mathrm{ar}), 6.20(\mathrm{~d}, 1 \mathrm{H}, \mathrm{NH}), 5.12(\mathrm{t}, 1 \mathrm{H}, J=9.6 \mathrm{~Hz}, \mathrm{H}-2)$, $5.03(\mathrm{t}, 1 \mathrm{H}, J=9.4 \mathrm{~Hz}, \mathrm{H}-3), 4.97(\mathrm{t}, 1 \mathrm{H}, J=9.2 \mathrm{~Hz}, \mathrm{H}-4), 4.31(\mathrm{t}$, $1 \mathrm{H}, J=9.9 \mathrm{~Hz}, \mathrm{H}-1), 4.12(\mathrm{dd}, 1 \mathrm{H}, J=5.1 \mathrm{~Hz}, \mathrm{H}-6), 4.02(\mathrm{dd}, 1 \mathrm{H}$, $J=11.8 \mathrm{~Hz}, \mathrm{H}-6^{\prime}$ ), 3.38 (ddd, $1 \mathrm{H}, J=2.8 \mathrm{~Hz}, \mathrm{H}-5$ ), 2.09, 2.05, 1.97, $1.95\left(4 \mathrm{~s}, 12 \mathrm{H}, \mathrm{CH}_{3} \mathrm{CO}\right) ;{ }^{13} \mathrm{C} \mathrm{NMR}\left(90 \mathrm{MHz}, \mathrm{CDCl}_{3}\right) \delta 170.4,170.1$, 170.0, 169.4 (4s, $\left.\mathrm{CH}_{3} \mathrm{CO}\right), 151.9(\mathrm{NHC}(=\mathrm{NOH}) \mathrm{Ar}), 148.7\left(\mathrm{CNO}_{2}\right)$, $136.5\left(\mathrm{~N}_{2} \mathrm{C}-\mathrm{C}\right), 129.5,123.6,82.5$ (C-1), 72.9, 72.5, 70.6, 68.3 (4s, C-2 C-3 C-4 C-5), 62.1 (C-6), 20.5, 20.6, 20.4 (3s, 4C, $\mathrm{CH}_{3} \mathrm{CO}$ ); Anal. Calcd for $\mathrm{C}_{21} \mathrm{H}_{25} \mathrm{~N}_{3} \mathrm{O}_{12}$ (511.45): C, 49.32; $\mathrm{H}, 4.93 ; \mathrm{N}, 8.22$. Found: $\mathrm{C}$, $50.02 ; \mathrm{H}, 5.03 ; \mathrm{N}, 8.16$.

\subsection{3. $N$-(2,3,4,6-Tetra-O-acetyl- $\beta$-D-glucopyranosyl)-4- cyanobenzamidoxime $8 \mathrm{c}$}

Prepared according to general procedure E to afford $8 \mathbf{c}$ (245 mg) in $18 \%$ yield.

Optimised synthesis of 8c: $2,3,4,6$-Tetra- $O$-acetyl- $\beta$-D-glucopyranosyl azide 7 ( $100 \mathrm{mg}, 0.26 \mathrm{mmol})$ was dissolved in dry dichloromethane $(1 \mathrm{~mL})$ and trimethyl phosphine $(0.268 \mathrm{~mL}, 1 \mathrm{M}$ in toluene) was added with stirring. During gas evolution, the reagent solution was freshly prepared by adding triethylamine to a solution of p-cyanophenyl hydroximinoyl chloride (153 mg, $0.26 \mathrm{mmol}$ ) in dry dichloromethane $(1 \mathrm{~mL})$ causing the formation of triethylamine hydrochloride precipitate. When TLC showed complete transformation of the starting material 7 ( $\sim 15 \mathrm{~min}$ ), the reagent solution was added to the mixture. After $10 \mathrm{~min}$, the reaction mixture was poured into water and was worked up as described in general procedure $\mathrm{E}$ to afford $8 \mathrm{c}(78 \mathrm{mg}, 60 \%$ yield).

$[\alpha]_{\mathrm{D}}+9.8\left(\mathrm{c} 0.2, \mathrm{CDCl}_{3}\right) ; \mathrm{mp}=120-123^{\circ} \mathrm{C} ;{ }^{1} \mathrm{H}$ NMR $(300 \mathrm{MHz}$, $\left.\left.\mathrm{CDCl}_{3}\right) \hat{\delta} 8.50(\mathrm{~s}, 1 \mathrm{H}, \mathrm{OH}), \widehat{7.71} \hat{\mathrm{d}}, 2 \mathrm{H}, J=8.6 \mathrm{~Hz}, \mathrm{H}-\mathrm{ar}\right), 7.65$ (d, $2 \mathrm{H}, J=8.3 \mathrm{~Hz}, \mathrm{H}-\mathrm{ar}), 6.16$ (d, $1 \mathrm{H}, J=10.4 \mathrm{~Hz}, \mathrm{NH}), 5.14(\mathrm{t}, 1 \mathrm{H}$, $J=9.4 \mathrm{~Hz}, \mathrm{H}-3$ or $\mathrm{H}-4), 5.03(\mathrm{t}, 1 \mathrm{H}, J=8.8 \mathrm{~Hz}, \mathrm{H}-2), 4.99(\mathrm{t}, 1 \mathrm{H}$, $J=9.4 \mathrm{~Hz}, \mathrm{H}-3$ or $\mathrm{H}-4), 4.31$ (dd, $1 \mathrm{H}, J=8.8 \mathrm{~Hz}, \mathrm{H}-1), 4.10(\mathrm{~d}, 1 \mathrm{H}$, $J=6.6 \mathrm{~Hz}, \mathrm{H}-6), 4.02$ (dd, $\left.1 \mathrm{H}, J=12.5 \mathrm{~Hz}, \mathrm{H}-6^{\prime}\right), 3.39$ (ddd, $1 \mathrm{H}$, $J=2.7 \mathrm{~Hz}, \mathrm{H}-5), 2.09,2.06,2.02,1.99\left(4 \mathrm{~s}, 12 \mathrm{H}, \mathrm{CH}_{3} \mathrm{CO}\right) ;{ }^{13} \mathrm{C} \mathrm{NMR}$ $\left(75 \mathrm{MHz}, \mathrm{CDCl}_{3}\right) \delta 170.4,170.2,170.0$ (3s, 4C, $\left.\mathrm{CH}_{3} \mathrm{CO}\right), 152.3$ $(\mathrm{NHC}=\mathrm{NOH}) \mathrm{Ar}), \quad 134.7 \quad\left(\mathrm{~N}_{2} \mathrm{C}-\mathrm{C}\right), 132.3,129.2,128.9(\mathrm{C}-\mathrm{CN})$, $113.9(\mathrm{CN}), 82.5$ (C-1), 72.9 (C-5), 72.4, 70.7, 68.3 (C-2 C-3 C-4), 62.1 (C-6), 21.0, 20.7, 20.6, $20.5\left(4 \mathrm{~s}, \mathrm{CH}_{3} \mathrm{CO}\right)$; Anal. Calcd for $\mathrm{C}_{22} \mathrm{H}_{25} \mathrm{~N}_{3} \mathrm{O}_{10}$ (491.46): C, 53.77; H, 5.13; N, 8.55. Found: $\widehat{C}, 53.43$; H, 5.09; N, 8.62.

4.6.4. $N$-(2,3,4,6-Tetra-O-acetyl- $\beta$-D-glucopyranosyl)-C-(2naphthyl)-amidoxime $8 \hat{\mathbf{h}}$

Prepared according to the general procedure E to afford $\mathbf{8 h}$ (92 $\mathrm{mg}$ ) in 6\% yield as a brownish foam which decomposed rapidly.

\subsection{Deacetylation of $\mathbf{N}$-glycosyl-amidoximes}

4.7.1. $N_{\text {- }}$ ( $\beta$-D-Glucopyranosyl)-4-nitrobenzamidoxime $9 \mathrm{~b}$

A few drops of NaOMe $(2.5 \mathrm{M}$ in $\mathrm{MeOH})$ were added to a solution of $\mathbf{8 b}(100 \mathrm{mg}, 0.19 \mathrm{mmol})$ in dry methanol $(7 \mathrm{~mL})$. The reaction mixture was stirred at $\mathrm{rt}$ for $5 \mathrm{~min}$ before the addition of cation exchange resin Amberlyst $15\left(\mathrm{H}^{+}\right.$form). The resin was filtered off and the solvent was removed. The residue was purified by chrystallization to give $56 \mathrm{mg}(83 \%)$ of $\mathbf{9 b}$.

$[\alpha]_{\mathrm{D}}-56$ (c 1.3, DMSO); $\mathrm{np}=165-168{ }^{\circ} \mathrm{C} ;{ }^{1} \mathrm{H}$ NMR $(300 \mathrm{MHz}$, DMSO-d $\left.d_{6}\right) \delta 10.39$ (s, $\left.1 \mathrm{H}, \mathrm{OH}\right), 8.22$ (d, 2H, $\left.J=8.4 \mathrm{~Hz}, \mathrm{H}-\mathrm{ar}\right), 7.91$ (s, 2H, J = 8.2 Hz, H-ar), 6.40 (d, 1H, J = 9.84 Hz, NH), 5.1-4.65 (m, $4 \mathrm{H}, \mathrm{OH}), 4.00-2.80$ (m, 7H, H-1 H-2 H-3 H-4 H-5 H-6 H-6'); ${ }^{13} \mathrm{C}$ NMR $\left(75 \mathrm{MHz}\right.$, DMSO-d $\left.d_{6}\right) \delta 152.0\left(\mathrm{CNO}_{2}\right), 147.6(\mathrm{~N}=\widehat{C N}), 138.5$ $\left(\mathrm{N}_{2} \mathrm{C}-\mathrm{C}\right), 129.6,123.1,83.8(\mathrm{C}-1), 78.1,77.3,72.8,70.1$ (4s, C-2 C3 C-4 C-5), 61.0 (C-6); Anal. Calcd for $\mathrm{C}_{13} \mathrm{H}_{17} \mathrm{~N}_{3} \mathrm{O}_{8}$ (343.30): 45.48; H, 4.99; N, 12.24. Found: C, 46.02; H, 5.25; N, 11.87.

\subsection{2. $N$-( $\beta$-D-Glucopyranosyl)-4-cyanobenzamidoxime 9c}

A few drops of NaOMe $(2.5 \mathrm{M}$ in $\mathrm{MeOH})$ were added to a solution of $8 c$ ( $90 \mathrm{mg}, 0.18 \mathrm{mmol})$ in dry methanol $(9 \mathrm{~mL})$. The reaction mixture was stirred at $\mathrm{rt}$ for $25 \mathrm{~min}$ before the addition of cation exchange resin Amberlyst $15\left(\mathrm{H}^{+}\right.$form). The resin was filtered off and the solvent was removed to give $48 \mathrm{mg}$ ( $81 \%$ ) of $9 \mathrm{c}$ as a syrup.

$[\alpha]_{\mathrm{D}}-132(c 0.6, \mathrm{MeOH}) ;{ }^{1} \mathrm{H} \mathrm{NMR}\left(360 \mathrm{MHz}, \mathrm{CD}_{3} \mathrm{OD}\right) \delta 7.65(\mathrm{~d}$, $2 \mathrm{H}, J=9.2 \mathrm{~Hz}, \mathrm{H}-\mathrm{ar}), 7.39$ (d, $2 \mathrm{H}, J=7.9 \mathrm{~Hz}, \mathrm{H}-\mathrm{ar}), 3.55(\mathrm{t}, 1 \mathrm{H}$, $J=11.8 \mathrm{~Hz}), \quad 3.40-3.10(\mathrm{~m}, 5 \mathrm{H}) ;{ }^{13} \mathrm{C} \mathrm{NMR}\left(90 \mathrm{MHz}, \mathrm{CD}_{3} \mathrm{OD}\right) \delta$ $156.4(\mathrm{~N}-\mathrm{C}=\mathrm{N}), 134.6\left(\mathrm{~N}_{2} \mathrm{C}-\mathrm{C}\right), 124.9,119.9(\mathrm{NC}-\mathrm{C}), 108.1(\mathrm{CN})$, 84.6 (C-1), 79.6, 78.6, 74.2, 71.5 (4s, C-2 C-3 C-4 C-5), 62.9 (C-6); Anal. Calcd for $\mathrm{C}_{14} \mathrm{H}_{17} \mathrm{~N}_{3} \mathrm{O}_{6}$ (323.31): ${ }_{\text {C. }}$ 52.01; H, 5.30; N, 13.00 . Found: C, 52.52; H, 5.23; N, 12.65.

\section{8. $N$-(Phenyl-nitrosomethylene)-2,3,4,6-tetra- $O$-acetyl- $\beta$-D- glucopyranosyl amine 10b}

Photolysis of $\mathbf{8 b}$ with a 60 Wheating lamp using 4 equiv of NBS in one portion: A solution of $\mathbf{8 b}(200 \mathrm{mg}, 0.39 \mathrm{mmol})$ in dry chloroform $(8 \mathrm{~mL})$ was placed in a $100 \mathrm{~mL}$ erlenmeyer flask equipped with a reflux condenser and NBS (278 $\mathrm{mg}, 1.56 \mathrm{mmol}$ ) was added in one portion. The mixture was irradiated with a $60 \mathrm{~W}$ white heating lamp from a distance of $1 \mathrm{~cm}$. After $15 \mathrm{~min}$, chloroform was added $(30 \mathrm{~mL})$, the mixture was washed with $5 \%$ aq $\mathrm{Na}_{2} \mathrm{SO}_{3}$, satd aq $\mathrm{NaHCO}_{3}$ and water. The organic layer was dried $\left(\mathrm{Na}_{2} \mathrm{SO}_{4}\right)$, filtered and the solvent was removed under vacuo. The residue was purified by column chromatography (EtOAc/hexane, 1:4) affording $\mathbf{1 0 b}$ as syrup in $30 \%$ yield $(58 \mathrm{mg})$. Traces of $\mathbf{1 2 b}(\sim 3 \%)$ were also detected in the crude product by NMR spectroscopy.

Compound 10b: $[\alpha]_{\mathrm{D}}+22\left(\right.$ c $0.4, \mathrm{CHCl}_{3}$ ); ${ }^{1} \mathrm{H}$ NMR $(300 \mathrm{MHz}$,

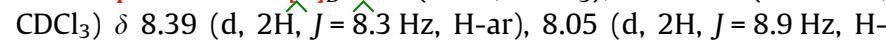
ar), $5.32(\mathrm{~d}, 1 \mathrm{H}, J=9.8 \mathrm{~Hz}, \mathrm{H}-1), 5.23(\mathrm{t}, 1 \mathrm{H}, J=9.5 \mathrm{~Hz}, \mathrm{H}-3), 4.83$ (t, $2 \mathrm{H}, J=9.4 \mathrm{~Hz}, \mathrm{H}-2 \mathrm{H}-4), 4.44$ (dd, $1 \mathrm{H}, J=12.9 \mathrm{~Hz}, \mathrm{H}-6), 4.13$ (dd, $1 \mathrm{H}, J=6.0 \mathrm{~Hz}, \mathrm{H}-6^{\prime}$ ), 3.90 (ddd, $1 \mathrm{H}, J=1.9 \mathrm{~Hz}, \mathrm{H}-5$ ), 2.16, 2.01, 1.99, $1.93\left(4 \mathrm{~s}, 12 \mathrm{H}, \mathrm{CH}_{3} \mathrm{CO}\right) ;{ }^{13} \mathrm{C}$ NMR $\left(75 \mathrm{MHz}, \mathrm{CDCl}_{3}\right) \delta$ $170.2,169.5,169.4,169.2\left(4 \mathrm{~s}, \mathrm{CH}_{3} \mathrm{CO}\right), 157.3\left(\mathrm{~N}_{2} \mathrm{C}-\mathrm{C}\right), 156.6$ $(\mathrm{NC}=\mathrm{N}), 150.1\left(\mathrm{CNO}_{2}\right), 131.5,123.7,82.0(\mathrm{C}-1), 75.3,72.2,68.7$, 66.8 (C-2 C-3 C-4 C-5), 61.0 (C-6), 20.7, 20.4, 20.3, 20.2 (4s, $\left.\mathrm{CH}_{3} \mathrm{CO}\right)$; Anal. Calcd for $\mathrm{C}_{21} \mathrm{H}_{23} \mathrm{~N}_{3} \mathrm{O}_{12}$ (509.43): C, 49.51; H, 4.55; N, 8.25. Found: C, 49.48; H, 4.76; N 8.22.

4.9. 3-(4-Nitrophenyl)-5-(D-gluco-1,2,3,5-tetraacetoxy-4hydroxypentyl)-1,2,4-oxadiazole 11b

Photolysis of $\mathbf{8 b}$ with a 375 Wheating lamp using 4 equiv of NBS in one portion: A solution of $\mathbf{8 b}(200 \mathrm{mg}, 0.39 \mathrm{mmol})$ in dry chloroform (12 mL) was placed in a $100 \mathrm{~mL}$ erlenmeyer flask equipped with a reflux condenser and NBS (278 mg, $1.56 \mathrm{mmol}, 4$ equiv) 
was added in one portion. The mixture was irradiated with a $375 \mathrm{~W}$ white heating lamp from a distance of $1 \mathrm{~cm}$. After $2 \mathrm{~h}$, the mixture was diluted with chloroform $(30 \mathrm{~mL})$, washed with $5 \%$ aq $\mathrm{Na}_{2} \mathrm{SO}_{3}$, satd aq $\mathrm{NaHCO}_{3}$ and water. The organic layer was dried $\left(\mathrm{Na}_{2} \mathrm{SO}_{4}\right)$, filtered and the solvent was removed under vacuo. The residue was purified by column chromatography (EtOAc/hexane, $1: 4)$ affording $\mathbf{1 2 b}(13 \mathrm{mg}, 13 \%)$ and $\mathbf{1 1 b}(20 \mathrm{mg}, 20 \%)$ as a syrup.

Compound 11b: $[\alpha]_{\mathrm{D}}+26.2\left(\right.$ c $\left.0.26, \mathrm{CHCl}_{3}\right) ;{ }^{1} \mathrm{H}$ NMR $(400 \mathrm{MHz}$, $\left.\mathrm{CDCl}_{3}\right) \delta 8.33(\mathrm{~d}, 2 \mathrm{H}, J=8.9 \mathrm{~Hz}, \mathrm{H}-\mathrm{ar}), 8.24(\mathrm{~d}, 2 \mathrm{H}, J=8.8 \mathrm{~Hz}, \mathrm{H}-$ ar), $6.16(\mathrm{~d}, 1 \mathrm{H}, J=6.7 \mathrm{~Hz}, \mathrm{H}-2), 5.73(\mathrm{dd}, 1 \mathrm{H}, J=3.1$ and $7.3 \mathrm{~Hz}$, $\mathrm{H}-4), 5.20-5.17(\mathrm{~m}, 1 \mathrm{H}, \mathrm{H}-5), 3.53(\mathrm{dd}, 1 \mathrm{H}, J=3.6$ and $11.9 \mathrm{~Hz}, \mathrm{H}-$ 6), 3.39 (dd, $\left.1 \mathrm{H}, J=6.4 \mathrm{~Hz}, \mathrm{H}-6^{\prime}\right), 2.18,2.11,2.10,2.08(4 \mathrm{~s}, 12 \mathrm{H}$, $\left.\mathrm{CH}_{3} \mathrm{CO}\right) ;{ }^{13} \mathrm{C}$ NMR $\left(100 \mathrm{MHz}, \mathrm{CDCl}_{3}\right.$ ) $\delta 174.3(\mathrm{C}-1), 169.5,169.4$, 169.3, $169.0\left(4 \mathrm{~s}, \mathrm{CH}_{3} \mathrm{CO}\right), 166.9(\mathrm{NC}=\mathrm{N}), 149.6\left(\mathrm{CNO}_{2}\right), 131.8\left(\mathrm{C}^{-}\right.$ $\left.\mathrm{CN}_{2}\right), 128.5,124.1,69.6,69.2,68.6$ (C-2 C-3 C-4), 65.7 (C-6), 30.2 (C-5), 20.6, 20.5, 20.3, $20.2\left(4 \mathrm{~s}, \mathrm{CH}_{3} \mathrm{CO}\right)$; $\mathrm{MS}(\mathrm{FAB}>0) \mathrm{m} / z=663.7$ $[\mathrm{M}+\text { dihydroxybenzoic acid }]^{+}$; Anal. Calcd for $\mathrm{C}_{21} \mathrm{H}_{23} \mathrm{~N}_{3} \mathrm{O}_{12}$ (509.43): C, 49.51; H, 4.55; N, 8.25. Found: C, 49.76, H 4.58, N 8.17.

\subsection{3-(4-Nitrophenyl)-5-(D-xylo-1,2,3,5-tetraacetoxy-4- oxopentyl)-1,2,4-oxadiazole $12 b$}

Photolysis of $\mathbf{8 b}$ with a 60 W heating lamp using 4 equiv of NBS in 4 portions: A solution of $\mathbf{8 b}(1 \mathrm{~g}, 1.96 \mathrm{mmol})$ in dry chloroform $(40 \mathrm{~mL})$ was placed in a $250 \mathrm{~mL}$ erlenmeyer flask equipped with a reflux condenser and NBS (348 mg, $1.96 \mathrm{mmol}$, 1 e्रquiv) was added. The mixture was irradiated with a $60 \mathrm{~W}$ white heating lamp from a distance of $1 \mathrm{~cm}$. After $30 \mathrm{~min}$, NBS (348 mg, $1.96 \mathrm{mmol}$, 1 equiv) was added to the reaction mixture. An additional 2 e्रquiv of NBS were added in two portions after $105 \mathrm{~min}$ and $135 \mathrm{~min}$, respectively. After $2 \mathrm{~h} 45 \mathrm{~min}$ total reaction time, the mixture was diluted with chloroform $(120 \mathrm{~mL})$, washed with $5 \%$ aq $\mathrm{Na}_{2} \mathrm{SO}_{3}$, satd aq $\mathrm{NaHCO}_{3}$ and water. The organic layer was dried $\left(\mathrm{Na}_{2} \mathrm{SO}_{4}\right)$, filtered and the solvent was removed under vacuo. The residue was purified by column chromatography (EtOAc/hexane, 1:4) affording $\mathbf{1 2 b}$ as a sole syrupy product in $45 \%$ yield $(447 \mathrm{mg})$.

Compound 12b: $[\alpha]_{\mathrm{D}}+19.4\left(c \quad 0.3, \mathrm{CHCl}_{3}\right) ;{ }^{1} \mathrm{H} \mathrm{NMR}(300 \mathrm{MHz}$, $\left.\mathrm{CDCl}_{3}\right) \delta 8.32(\mathrm{~d}, 2 \mathrm{H}, J=10.1 \mathrm{~Hz}, \mathrm{H}-\mathrm{ar}), 8.22(\mathrm{~d}, 2 \mathrm{H}, J=9.1 \mathrm{~Hz}, \mathrm{H}-$ ar), $6.27(\mathrm{~d}, 1 \mathrm{H}, J=5.9 \mathrm{~Hz}, \mathrm{H}-2), 5.90(\mathrm{dd}, 1 \mathrm{H}, J=3.7 \mathrm{~Hz}, \mathrm{H}-3)$, 5.54 (d, 1H, H-4), 4.87 (d, $1 \mathrm{H}, J=17.3 \mathrm{~Hz}, \mathrm{H}-6), 4.72$ (d, 1H, H-6'), 2.19, 2.13, 2.12, $2.01\left(4 \mathrm{~s}, 12 \mathrm{H}, \mathrm{CH}_{3} \mathrm{CO}\right) ;{ }^{13} \mathrm{C} \mathrm{NMR}\left(75 \mathrm{MHz}, \mathrm{CDCl}_{3}\right)$ $\delta$ 197.1 (C-5), 174.3 (C-1), 169.7, 169.4, 169.2, 168.9 (4s, $\left.\mathrm{CH}_{3} \mathrm{CO}\right)$, $167.0\left(\mathrm{~N}_{2} \mathrm{CAr}\right), 149.6\left(\mathrm{CNO}_{2}\right), 131.7\left(\mathrm{~N}_{2} \mathrm{C}-\mathrm{C}\right), 128.5,124.1,73.7(\mathrm{C}-$ 3), 69.5 (C-4), 66.4 (C-6), 65.4 (C-2), 23.1, 20.2, 20.1 (3s, 4C, $\left.\mathrm{CH}_{3} \mathrm{CO}\right)$; $\mathrm{MS}(\mathrm{FAB}>0) \quad m / z=508[\mathrm{M}+\mathrm{H}]^{+}$; Anal. Calcd for $\mathrm{C}_{21} \mathrm{H}_{21} \mathrm{~N}_{3} \mathrm{O}_{12}$ (507.41): C, 49.71; H, 4.17; N, 8.28. Found: C, 49.58; $\mathrm{H}, 4.23 ; \mathrm{N}, 8.24$.

\subsection{1. $(1 R, 2 S, 3 R, 4 R)-1,2,3,5$-Tetra-O-benzyl-1-C-[3-(4-methoxy- phenyl)-4N-oxido-1,2,4-oxadiazole-5-yl]pentitol 15}

A solution of $\mathrm{Et}_{3} \mathrm{~N}(240 \mu \mathrm{L}, 1.72 \mathrm{mmol}, 12$ equiv) in toluene $(2 \mathrm{~mL}$ ) was added dropwise over $8 \mathrm{~h}$ (syringe pump) to a solution of hydroximolactone $\mathbf{1 3}(80 \mathrm{mg}, 0.14 \mathrm{mmol}$ ) and $\alpha$-chloro-p-methoxybenzaldoxime ( $210 \mathrm{mg}, 1.15 \mathrm{mmol}, 8$ equiv) in refluxing toluene $(15 \mathrm{~mL})$. After $24 \mathrm{~h}$ reflux, TLC showed a partial conversion of 13 into several products. After concentration, the residue was purified by column chromatography (PE/EtOAc, 7:3) to afford unreacted 13 (33 mg) then 15 (13 mg, 13\% isolated yield). Based on the converted starting material, the calculated yield was $22 \%$.

Compound 15: $R_{\mathrm{f}}=0.55$ (EP/EtOAc, 7:3); ${ }^{1} \mathrm{H}$ NMR $(500 \mathrm{MHz}$, $\left.\mathrm{CDCl}_{3}\right) \delta 7.15-7.38$ (m, 22H, H-ar), 6.88 (d, $2 \mathrm{H}, J=8.7 \mathrm{~Hz}, \mathrm{H}-\mathrm{ar}$ ), 4.75 (d, 个H, $J=12.2 \mathrm{~Hz}, \mathrm{CH}_{2} \mathrm{Ph}$ ), 4.66 (m, 2H, H-5 CH $\left.\mathrm{CH}_{2} \mathrm{Ph}\right), 4.59$ (d, $1 \mathrm{H}, J=12.4 \mathrm{~Hz}, \mathrm{CH}_{2} \mathrm{Ph}$ ), 4.55 (m, 3H, $\mathrm{CH}_{2} \mathrm{Ph}$ ), 4.47 (br s, $1 \mathrm{H}, \mathrm{CH}_{2} \mathrm{Ph}$ ), $4.41\left(\mathrm{~d}, 1 \mathrm{H}, \mathrm{J}=11.8 \mathrm{~Hz}, \mathrm{CH}_{2} \mathrm{Ph}\right), 4.18$ (br s, $\left.1 \mathrm{H}, \mathrm{H}-2\right), 3.96(\mathrm{~m}, 1 \mathrm{H}, \mathrm{H}-$ 3), 3.84-3.88 (m, 2H, H-4 H-6), 3.79 (s, $\left.4 \mathrm{H}, \mathrm{H}-6 \mathrm{OCH}_{3}\right) ;{ }^{13} \mathrm{C} \mathrm{NMR}$ $\left(125 \mathrm{MHz}, \mathrm{CDCl}_{3}\right) \delta 156.3,154.2(\mathrm{NCO}), 140.8(\mathrm{NC}=\mathrm{N}), 137.8$, 137.4, 137.0, 136.6, 130.1, 127.0-128.6 (m, 16C, CH-ar), 121.4 (s, 2C, CH-ar), 114.2 (s, 2C, CH-ar), 80.8 (C-3), 77.2 (C-4), 77.1 (C-5), $73.4\left(\mathrm{CH}_{2} \mathrm{Ph}\right), 73.0\left(\mathrm{CH}_{2} \mathrm{Ph}\right), 72.9(\mathrm{C}-2), 71.7\left(\mathrm{CH}_{2} \mathrm{Ph}\right), 71.0\left(\mathrm{CH}_{2} \mathrm{Ph}\right)$, $67.7(\mathrm{C}-6), 55.5\left(\mathrm{OCH}_{3}\right)$; MS (ESI $\left.>0\right) \mathrm{m} / \mathrm{z}=703.0[\mathrm{M}+\mathrm{H}]^{+}, 725.0$ $[\mathrm{M}+\mathrm{Na}]^{+}, 1404.9[2 \mathrm{M}+\mathrm{H}]^{+}, 1426.9[2 \mathrm{M}+\mathrm{Na}]^{+}$; HRMS $(\mathrm{ESI}>0) \mathrm{ml}$ $z=\mathrm{C}_{42} \mathrm{H}_{43} \mathrm{~N}_{2} \mathrm{O}_{8}[\mathrm{~N} \mathrm{M}+\mathrm{H}]^{+}$calcd 703.3019; found 703.3022.

\subsection{Enzymatic methods}

Glycogen phosphorylase $b$ was prepared from rabbit skeletal muscle according to the method of Fischer and Krebs, ${ }^{45}$ using dithiothreitol instead of L-cysteine, and recrystallized at least three times before use. Kinetic experiments were performed in the direction of glycogen synthesis as described previously. ${ }^{18}$ Kinetic data for the inhibition of rabbit skeletal muscle glycogen phosphorylase were collected using different concentrations of $\alpha$-D-glucose-1phosphate $(2-20 \mathrm{mM})$, constant concentrations of glycogen $(1 \%$ $\mathrm{w} / \mathrm{v}$ ) and AMP (1 mM), and various concentrations of inhibitors. Inhibitors were dissolved in dimethyl sulfoxide (DMSO) and diluted in the assay buffer $(50 \mathrm{mM}$ triethanolamine, $1 \mathrm{mM}$ EDTA and $1 \mathrm{mM}$ dithiothreitol) so that the DMSO concentration in the assay should be lower than $1 \%$. The enzymatic activities were presented in the form of double-reciprocal plots (Lineweaver-Burk) applying a nonlinear data analysis program. The inhibitor constants $\left(K_{\mathrm{i}}\right)$ were determined by Dixon plots, by replotting the slopes from the Lineweaver-Burk plots against the inhibitor concentrations. ${ }^{26,30}$ The means of standard errors for all calculated kinetic parameters averaged to less than $10 \%$.

\section{Acknowledgements}

Support of our bilateral collaboration by the French Ministry of Foreign Affairs (PAI Balaton projects 03767RJ and 11061PE), by the CNRS (Project No. 7885 and PICS No.4576), as well as support by Région Rhône-Alpes are gratefully acknowledged. The synthetic work in Debrecen was supported by the Hungarian Scientific Research Fund (OTKA 46081 and 61336). V.N. thanks the French Embassy in Budapest for financial support of her co-tutored PhD thesis. M.B. thanks the Région Rhone-Alpes for financing his co-tutored PhD thesis (MIRA Mobilité).

\section{Supplementary data}

Supplementary data (attempted preparations of glucopyranosylidene-spiro-oxadiazolines, separation of amidoximes, structural elucidation of amidoximes and oxidation products) associated with this article can be found, in the online version, at doi:10.1016/j.bmc.2009.05.080.

\section{References and notes}

1. Ross, S. A.; Gulve, E. A.; Wang, M. H. Chem. Rev. 2004, 104, 1255-1282.

2. Diamond, J. Nature 2003, 423, 599-602.

3. Zimmet, P.; Alberti, K. G. M. M.; Shaw, J. Nature 2001, 414, 782-861.

4. Alberti, G.; Zimmet, P.; Shaw, J.; Bloomgarden, Z. T.; Kaufman, F.; Silink, M. Diabetes Care 2004, 27, 1798-1811.

5. Bloomgarden, Z. T. Diabetes Care 2004, 27, 998-1010.

6. Ehtisham, S.; Barrett, T. G. Ann. Clin. Biochem. 2004, 41, 10-16.

7. Wagman, A. S.; Nuss, J. M. Curr. Pharm. Des. 2001, 7, 417-450.

8. Kurukulasuriya, R.; Link, J. T.; Madar, D. J.; Pei, Z.; Richards, S. J.; Rohde, J. J.; Souers, A. J.; Szczepankiewicz, B. G. Curr. Med. Chem. 2003, 10, 123-153.

9. McCormack, J. G.; Westergaard, N.; Kristiansen, M.; Brand, C. L.; Lau, J. Curr. Pharm. Des. 2001, 7, 1451-1474.

10. Oikonomakos, N. G. Curr. Protein Pept. Sci. 2002, 3, 561-586.

11. Treadway, J. L.; Mendys, P.; Hoover, D. J. Exp. Opin. Invest. Drugs 2001, 10, 439454.

12. Somsák, L.; Czifrák, K.; Tóth, M.; Bokor, É.; Chrysina, E. D.; Alexacou, K. M.; Hayes, J. M.; Tiraidis, C.; Lazoura, E.; Leonidas, D. D.; Zographos, S. E.; Oikonomakos, N. G. Curr. Med. Chem. 2008, 15, 2933-2983. 
1090 13. Oikonomakos, N. G.; Somsák, L. Curr. Opin. Invest. Drug 2008, 9, 379-395.

14. Somsák, L.; Nagy, V.; Hadady, Z.; Docsa, T.; Gergely, P. Curr. Pharm. Des. 2003, 9, 1177-1189.

15. Somsák, L.; Nagy, V.; Hadady, Z.; Felföldi, N.; Docsa, T.; Gergely, P. Recent developments in the synthesis and evaluation of glucose analog inhibitors of glycogen phosphorylases as potential antidiabetic agents. In Frontiers in Medicinal Chemistry; Reitz, A. B., Kordik, C. P., Choudhary, M. I., Rahman, A. u., Eds.; Bentham, 2005; Vol. 2, pp 253-272.

16. Watson, K. A.; Mitchell, E. P.; Johnson, L. N.; Cruciani, G.; Son, J. C.; Bichard, C. J. F.; Fleet, G. W. J.; Oikonomakos, N. G.; Kontou, M.; Zographos, S. E. Acta Crystallograph 1995, D51, 458-472.

17. Gregoriou, M.; Noble, M. E. M.; Watson, K. A.; Garman, E. F.; Krülle, T. M.; Fuente, C.; Fleet, G. W. J.; Oikonomakos, N. G.; Johnson, L. N. Protein Sci. 1998, 7, 915-927.

18. Oikonomakos, N. G.; Skamnaki, V. T.; Õsz, E.; Szilágyi, L.; Somsák, L.; Docsa, T.; Tóth, B.; Gergely, P. Bioorg. Med. Chem. 2002, 10, 261-268.

19. Nagy, V.; Felföldi, N.; Praly, J.-P.; Docsa, T.; Gergely, P.; Chrysina, E. D.; Tiraidis, C.; Alexacou, K. M.; Leonidas, D. D.; Zographos, S. E.; Oikonomakos, N. G.; Somsák, L. in preparation.

20. Chrysina, E. D.; Nagy, V.; Felföldi, N.; Telepó, K.; Praly, J.-P.; Docsa, T.; Gergely, P.; Alexacou, K. M.; Leonidas, D. D.; Zographos, S. E.; Oikonomakos, N. G.; Somsák, L. in preparation.

21. Elek, R.; Kiss, L.; Praly, J.-P.; Somsak, L. Carbohydr. Res. 2005, 340, 1397-1402.

22. Praly, J.-P.; Faure, R.; Joseph, B.; Kiss, L.; Rollin, P. Tetrahedron 1994, 50, 65596568.

23. Somsák, L.; Nagy, V.; Vidal, S.; Czifrák, K.; Berzsényi, E.; Praly, J.-P. Bioorg. Med. Chem. Lett. 2008, 18, 5680-5683.

24. Anagnostou, E.; Kosmopoulou, M. N.; Chrysina, E. D.; Leonidas, D. D.; Hadjiloi, T.; Tiraidis, C.; Zographos, S. E.; Györgydeák, Z.; Somsák, L.; Docsa, T.; Gergely, P.; Kolisise, F. N.; Oikonomakos, N. G. Bioorg. Med. Chem. 2006, 14, 181-189.

25. Petsalakis, E. I.; Chrysina, E. D.; Tiraidis, C.; Hadjiloi, T.; Leonidas, D. D.; Oikonomakos, N. G.; Aich, U.; Varghese, B.; Loganathan, D. Bioorg. Med. Chem. 2006, 14, 5316-5324.

26. Somsák, L.; Kovács, L.; Tóth, M.; Õsz, E.; Szilágyi, L.; Györgydeák, Z.; Dinya, Z.; Docsa, T.; Tóth, B.; Gergely, P. J. Med. Chem. 2001, 44, 2843-2848.
27. Györgydeák, Z.; Hadady, Z.; Felföldi, N.; Krakomperger, A.; Nagy, V.; Tóth, M.; Brunyánszky, A.; Docsa, T.; Gergely, P.; Somsák, L. Bioorg. Med. Chem. 2004, 12, 4861-4870.

28. Bichard, C. J. F.; Mitchell, E. P.; Wormald, M. R.; Watson, K. A.; Johnson, L. N.; Zographos, S. E.; Koutra, D. D.; Oikonomakos, N. G.; Fleet, G. W. J. Tetrahedron Lett. 1995, 36, 2145-2148.

29. Krülle, T. M.; Fuente, C.; Watson, K. A.; Gregoriou, M.; Johnson, L. N.; Tsitsanou, K. E.; Zographos, S. E.; Oikonomakos, N. G.; Fleet, G. W. J. Synlett 1997, 211-213.

30. Õsz, E.; Somsák, L.; Szilágyi, L.; Kovács, L.; Docsa, T.; Tóth, B.; Gergely, P. Bioorg. Med. Chem. Lett. 1999, 9, 1385-1390.

31. Oikonomakos, N. G.; Kosmopoulou, M. N.; Zographos, S. E.; Leonidas, D. D.; Somsák, L.; Nagy, V.; Praly, J.-P.; Docsa, T.; Tóth, B.; Gergely, P. Eur. J. Biochem. 2002, 269, 1684-1696.

32. Brochard, L.; Joseph, B.; Viaud, M.-C.; Rollin, P. Synth. Commun. 1994, 24, 1403 1414.

33. Praly, J.-P.; Descotes, G. Tetrahedron Lett. 1990, 31, 1133-1136.

34. Cekovic, Z. Tetrahedron 2003, 59, 8073-8090.

35. Descotes, G. J. Carbohydr. Chem. 1988, 7, 1-20.

36. Eloy, F.; Lenaers, R. Chem. Rev. 1962, 62, 155-183.

37. Kovács, L.; Osz, E.; Domokos, V.; Holzer, W.; Györgydeák, Z. Tetrahedron 2001, 57, 4609-4621.

38. Werner, A.; Bloch, C. Chem. Ber. 1899, 32, 1975-1985.

39. Werner, A.; Buss, H. Chem. Ber. 1894, 27, 2193-2201.

40. Liu, K.-C.; Shelton, B. R.; Howe, R. K. J. Org. Chem. 1980, 45, 3916-3918.

41. Aebischer, B. M.; Hanssen, H. W.; Vasella, A. T.; Schweizer, W. B. J. Chem. Soc., Perkin Trans. 1 1982, 2139-2147.

42. Beer, D.; Vasella, A. Helv. Chim. Acta 1985, 68, 2254-2274.

43. Quadrelli, P.; Invernizzi, A. G.; Falzoni, M.; Caramella, P. Tetrahedron 1997, 53, 1787-1796.

44. Quadrelli, P.; Caramella, P. Curr. Org. Chem. 2007, 11, 959-986.

45. Fischer, E. H.; Krebs, E. G.; Colowick, S. P.; Kaplan, N. O.. [49a] Muscle phosphorylase $b$ : $x$ Glucose-1-phosphate+ $G n<->G n+x+x$ inorganic phosphate (where Gn designates glycogen containing $n$ glucose residues). In Methods in Enzymology; Academic Press, 1962; vol. 5. pp 369-373. 